\title{
Zinc protoporphyrin binding to telomerase complexes and inhibition of telomerase activity*
}

\author{
Zhaowen Zhu ${ }^{1,2}$ | Huy Tran ${ }^{2}$ | Meleah M. Mathahs ${ }^{1}$ | Brian D. Fink ${ }^{1}$ | John A. Albert ${ }^{1}$ | \\ Thomas O. Moninger $^{3}$ | Jeffery L. Meier ${ }^{1,2} \mid$ Ming Li $^{1}$ | Warren N.Schmidt ${ }^{1,2}$ (1)
}

${ }^{1}$ Department of Internal Medicine and Research Service, Veterans Affairs Medical Center, lowa City, lowa, USA

${ }^{2}$ Department of Internal Medicine, Roy G. and Lucille A. Carver College of Medicine, University of lowa, lowa City, lowa, USA

${ }^{3}$ Central Microscopy Research Facility Roy G. and Lucille A. Carver College of Medicine, University of lowa, lowa City, lowa, USA

\section{Correspondence}

Warren N. Schmidt, Professor, Division of $\mathrm{Gl} /$ Hepatology, Department of Internal Medicine, University of lowa College of Medicine, Staff Physician GI/Hepatology, Veterans Affairs Medical Center, lowa City, IA 52242, USA.

Email:warren-schmidt@uiowa.edu

Funding information

Warren N. Schmidt, Grant/Award

Number: 2101BX000159-09

\begin{abstract}
Zinc protoporphyrin (ZnPP), a naturally occurring metalloprotoporphyrin (MPP), is currently under development as a chemotherapeutic agent although its mechanism is unclear. When tested against other MPPs, ZnPP was the most effective DNA synthesis and cellular proliferation inhibitor while promoting apoptosis in telomerase positive but not telomerase negative cells. Concurrently, ZnPP down-regulated telomerase expression and was the best overall inhibitor of telomerase activity in intact cells and cellular extracts with $\mathrm{IC}_{50}$ and $\mathrm{EC}_{50}$ values of ca 2.5 and $6 \mu \mathrm{M}$, respectively. The natural fluorescence properties of ZnPP enabled direct imaging in cellular fractions using non-denaturing agarose gel electrophoresis, western blots, and confocal fluorescence microscopy. ZnPP localized to large cellular complexes (>600 kD) that contained telomerase and dysskerin as confirmed with immunocomplex mobility shift, immunoprecipitation, and immunoblot analyses. Confocal fluorescence studies showed that ZnPP co-localized with telomerase reverse transcriptase (TERT) and telomeres in the nucleus of synchronized S-phase cells. Zn PP also co-localized with TERT in the perinuclear regions of log phase cells but did not co-localize with telomeres on the ends of metaphase chromosomes, a site known to be devoid of telomerase complexes. Overall, these results suggest that $\mathrm{ZnPP}$ does not bind to telomeric sequences per se, but alternatively, interacts with other structural components of the telomerase complex to inhibit telomerase activity. In conclusion, ZnPP actively interferes with telomerase activity in neoplastic cells, thus promoting pro-apoptotic and anti-proliferative properties. These data support further development of natural or synthetic protoporphyrins for use as chemotherapeutic agents to augment current treatment protocols for neoplastic disease.
\end{abstract}

Abbreviations: ANOVA, analysis of variance; CoPP, cobalt protoporphyrin; FePP, iron protoporphyrin (heme); MPP, metalloprotoporphyrin [Fe, Zn, Sn, Co]; MTT, 3-(4,5-dimethylthiazol2-yl)-2,5-diphenyltetrazolium bromide; NS, nonstructural replicon of HCV; RT-PCR, reverse transcription - polymerase chain reaction; SnPP, tin protoporphyrin; SYBR, N', N'-dimethyl$\mathrm{N}$-[4-[(E)-(3-methyl-1,3-benzothiazol-2-ylidene)methyl]-1- phenylquinolin-1-ium-2-yl]-N-propylpropane-1,3-diamine; TERC, telomerase RNA component; TERT, telomerase reverse transcriptase; TMPyP4, tetra-(N-methyl-4-pyridyl)porphyrin; TRAP, telomerase repeat amplification protocol; WB, western blot; ZnPP, zinc protoporphyrin.

*Supported by VA Merit grant (2101BX000159-09) to W.N.S. and VA Merit grant (I01 BX004434) to J.L.M.

This is an open access article under the terms of the Creative Commons Attribution License, which permits use, distribution and reproduction in any medium, provided the original work is properly cited.

(c) 2021 The Authors. Pharmacology Research \& Perspectives published by British Pharmacological Society and American Society for Pharmacology and Experimental Therapeutics and John Wiley \& Sons Ltd. 
KEYWORDS

cancer chemotherapy, telomerase enzymatic activity, telomerase reverse transcriptase,

telomere, zinc protoporphyrin

\section{1 | INTRODUCTION}

Telomerase is a cellular reverse transcriptase that is reactivated in about $85 \%$ of all cancers. ${ }^{1}$ The enzyme maintains adequate lengths of chromosomal 3' DNA telomeric strand ends, which are continuous sequences of -(TTAGGG)n-- that progressively shorten with each replication cycle because of the DNA polymerase 3 ' end replication problem. Using an RNA template, telomerase adds complementary DNA bases to the 3' telomere end which prevents chromosomal end damage and enables prolonged cellular proliferation, the hallmark of cancer cells. In rapidly dividing malignant cells, telomeres need constant repair to enable high replication rates. ${ }^{2}$ This activity is so crucial to malignancy that even in the $15 \%$ of neoplastic cells that do not express telomerase, telomeric ends are maintained by an alternative recombination process. ${ }^{3}$

Considerable evidence supports the feasibility of telomerase inhibitors as chemotherapeutic agents ${ }^{4-6}$ for a number of neoplastic diseases. As a class, planar, positively charged polyaromatic compounds such as porphyrins have been shown to have anti-telomerase activity. ${ }^{7}$ Porphyrins are known to bind and stabilize single-stranded telomeric DNA sequences at guanine secondary structures known as quadruplexes (G4) ${ }^{8}$ and impact telomerase presumably through substrate inhibition. ${ }^{7,9,10}$

Metalloprotoporphyrins (MPP) such as FePP and ZnPP, represent a subclass of important naturally occurring porphyrins that are also known to bind G4 structures in general ${ }^{11}$ and some telomeric sequences specifically. ${ }^{12,13}$ Additionally, ZnPP and conjugated derivatives such as ZnPP-polyethylene glycol have been widely studied in experimental rodent systems for use as chemotherapeutic agents. ${ }^{14,15}$ Considering the widespread interest in porphyrins as telomerase inhibitors as well as work with ZnPP as a chemotherapeutic agent, it is surprising that no studies have addressed potential interactions of ZnPP and other MPPs with telomerase.

The aim of the present study was to determine whether common MPPs impact telomerase expression and enzymatic activity in established hepatoma cells. We show that ZnPP abruptly halts DNA synthesis and promotes apoptosis, while concomitantly depressing the expression of telomerase as well as other proliferative proteins such as cyclin D1 and $\beta$-catenin. Furthermore, ZnPP effectively inhibits telomerase activity in intact cells, crude cellular lysates, and immunoprecipitates (IP) while localizing to large protein complexes that contain telomerase. The effects of ZnPP on apoptosis and toxicity were observed primarily in cells that contain telomerase, thus directly implicating a role for telomerase inhibition in the chemotherapeutic activities of ZnPP. These data indicate that ZnPP and perhaps other natural or synthetic MPPs should be studied further for use as chemotherapeutic agents in a wide range of telomerase positive neoplasms.

\section{2 | MATERIALS AND METHODS}

\section{1 | Materials}

Taq DNA polymerase (Perkin-Elmer Cetus, Norwalk, CT), and Moloney murine leukemia virus reverse transcriptase (Gibco/BRL Life Technologies, Gaithersburg, MD) were used in these studies. Electrophoresis supplies were purchased from Bio-Rad (CA).

All MPPs were obtained from Frontier Scientific, Inc (Logan, UT) and were $>97 \%$ purity (ZnPP Zn625-9, SnPP Sn749-9, CoPP Co654-9 and FePP H651-9). For MPP structures, see Figure S1. MPPs were dissolved in minimal volumes of dimethyl sulfoxide (DMSO) and diluted into culture media or assay buffers to achieve the final concentration. Controls received an identical volume of diluted solvent only. BIBR1532 was obtained from Cayman Chemical (Ann Arbor, MI. Item No. 16608). Colcemid was purchased from Roche Diagnostics $\mathrm{GmbH}$ (Mannheim, Germany. Cat. No. 10295892001).

$\alpha^{-32} \mathrm{P}-\mathrm{dGTP}(6000 \mathrm{Ci} / \mathrm{mmol})$ was obtained from Perkin-Elmer (Waltham, MA. \#BLU514Z). ${ }^{3} \mathrm{H}$-thymidine $(86 \mathrm{Ci} / \mathrm{mM}$ ) was from Amersham (Little Chalfont, U.K. TRK-758).

\subsection{Antibodies and probes}

See Table 1.

\section{3 $\quad$ Cell lines and cell culture}

Huh7, Hek293, and the HCV permissive clonal line Huh7.5 cells were maintained in routine cultures as described. ${ }^{16}$ The human hepatoma cell line (Huh5.15) with replicating sub-genomic HCV RNA (genotype 1b) (Huh5.15NS) ${ }^{17}$ was cultivated as described. ${ }^{18}$ Wild type Hek293 cells were obtained from University of lowa Tissue Culture stocks and passed routinely in minimal essential medium containing $10 \%$ fetal bovine serum. Telomerase negative U2OS osteosarcoma cells were obtained from American Type Culture Collection and passed using recommended media conditions. U2OS extracts were routinely tested by immunoblot analysis to ensure TERT negativity.

\subsection{Vectors and constructs}

Lipofectamine $^{T \mathrm{M}} 2000$ (ThermoFisher) was used for all transfections and closely followed the manufacturer's protocol. For TERT overexpression the catalytically active TERT plasmid $\mathrm{pCI}$ neo-hEST2, a gift from Dr. Robert Weinberg (Addgene plasmid \# 1781) ${ }^{19}$ was used. 
TABLE 1 List of antibodies, sources, and working dilutions

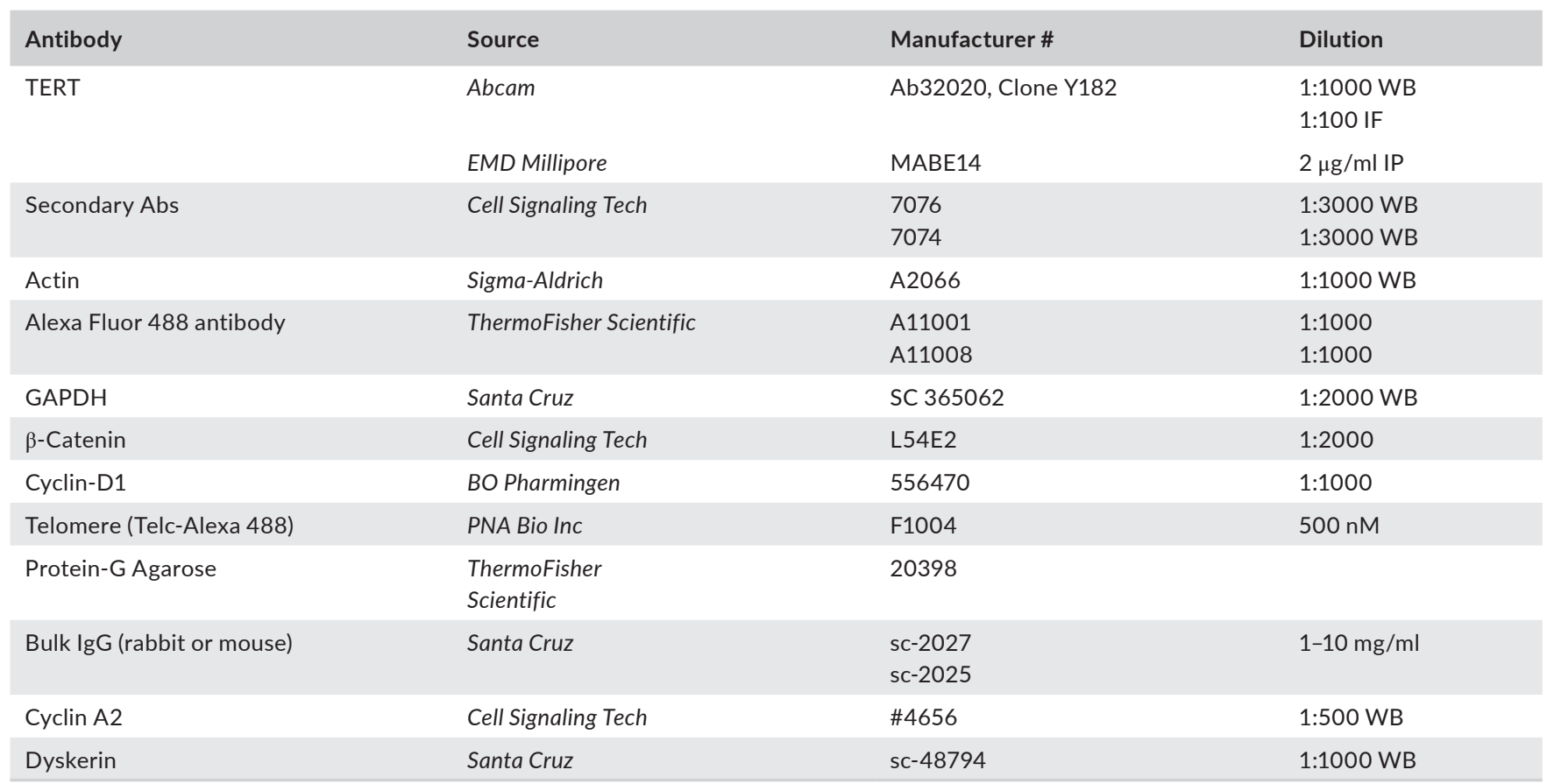

Note: Antibodies and immunological probes listed in the text were collated for reader convenience and easy access. Dilutions reflect titers used for assays as described further in the text.

Telomerase RNA component (TERC) plasmid (pBS U3-hTR-500) was also obtained from Addgene (plasmid \# 28170), a gift from Dr. Kathleen Collins. ${ }^{20,21}$

\subsection{DNA synthesis, cellular proliferation and apoptosis}

\subsection{1 | DNA synthesis}

Semi-quantitative differences in DNA synthesis were determined by measuring ${ }^{3} \mathrm{H}$-thymidine uptake into whole cells in log phase growth. At indicated time points, cell cultures were washed with serum-free, thymidine deficient medium, then incubated with fresh medium containing $1 \mu \mathrm{Ci} / \mathrm{ml}$ of ${ }^{3} \mathrm{H}$-thymidine for two hours at $37^{\circ} \mathrm{C}$. Cultures were washed twice with PBS while attached to dishes, then lysed with $0.5 \mathrm{M} \mathrm{NaOH}$ and the relative amount of ${ }^{3} \mathrm{H}$-thymidine quantified by scintillation counting.

\subsection{2 | Cell proliferation}

MPPs were tested for effects on cell proliferation and viability using MTT (3-(4,5-dimethylthiazol-2-yl)-2,5-diphenyltetrazolium bromide) dye conversion assay (Cell Titer 96, Promega) as we described ${ }^{22}$ with some modifications. Cells were plated into 96 well plates and allowed to attach overnight. MPPs were added to the cultures and then incubated for the times indicated. At assay time, MTT reagent was added and absorbance measured at $570 \mathrm{~nm}$. Controls included buffer blanks containing MPP because of background absorbances reported for MPPs by us ${ }^{22}$ and others. ${ }^{23}$ The formula used for determination of viable cells relative to controls was:

$\%$ viable cells $=\left(a_{b s_{\text {sample }}}-\mathrm{abs}_{\text {blank }}\right) /\left(a b s_{\text {conrtrol }}-\mathrm{abs}_{\text {blank }}\right) \times 100$

Cell viability was also directly determined with trypan blue staining as previously described and closely correlated with MTT assay. ${ }^{24}$

\subsection{3 | Apoptosis assay}

For detection of MPP apoptotic effects, cells were treated with MPPs (0, 2.5, 5 and $10 \mu \mathrm{M})$ for $48 \mathrm{~h}$ and assayed using PE Annexin V Apoptosis Detection Kit (Cat. No. 559763, BD Biosciences) as recommended by the manufacturer.

\subsection{Quantification of telomerase activity by real- time quantitative PCR and TRAP assay}

Telomerase reverse transcriptase (TERT) enzymatic activity was determined using Telomeric Repeat Amplification Protocol (TRAP) with Real-time quantification as described previously, ${ }^{25}$ or measured directly using $\alpha^{-32} \mathrm{P}$-dGTP incorporation as described ${ }^{26}$ with modifications as below.

For TRAP assay, a Quantitative Telomerase Detection Kit (US Biomax, Inc) was used to assay cellular lysates or TERT immunoprecipitates (IP) according to the manufacturer's directions. Relative 
telomerase activity was derived from a standard curve of reference samples and data were analyzed using relative fluorescence units as compared to controls. In some cases, telomerase reaction products were visualized and quantified with TRAPeze system (EMD/Millipore). After separation of the DNA products using $10 \%$ non-denaturing PAGE and SYBR fluorescence labeling [1x SYBR safe DNA gel stain (Invitrogen)], gel bands were imaged with iBright 1500 (Invitrogen). The intensity of the sample's TRAP ladder and internal control was first measured using GelAnalyzer [GelAnalyzer 19.1 (www.gelanalyzer. com)] as recommended. Then, the relative telomerase activity was determined by the ratio of the intensity of the sample's TRAP ladder (telomerase products, TP) to that of the internal control (IC) band.

\subsection{Direct telomerase activity assay}

For direct telomerase activity assay, a modified procedure of Tomlinson et $\mathrm{al}^{26}$ was used. Briefly, HEK-293T cell pellet from $10^{7}$ cells overexpressing TERT, TERC, and dyskerin was obtained from Abbexa Ltd (UK. Abx069991). The whole-cell lysate was produced using $1 \mathrm{ml}$ buffer $\mathrm{A}$ [20 mM HEPES-KOH buffer $(\mathrm{pH} 8), 300 \mathrm{mM}$ $\mathrm{KCl}, 2 \mathrm{mM} \mathrm{MgCl}_{2}, 0.1 \% \mathrm{v} / \mathrm{v}$ Triton X-100, 10\% v/v glycerol]. Immunoprecipitation of telomerase was performed with anti-hTERT polyclonal sheep antibody (abx120550, Abbexa, U.K.). The telomerase was finally eluted with hTERT peptide (abx069990, Abbexa, U.K.). $10 \mu \mathrm{l}$ of eluates were mixed with different concentrations of MPP and incubated on ice for $2 \mathrm{~h}$. Then the pellet-MPP complex was added to the extension reaction mixture composed of $1 x$ telomerase buffer, $300 \mathrm{mM} \mathrm{KCl}, 1 \mu \mathrm{M}$ Bio-L-18GGG, 1 mM dATP, 1 mM dTTP, $10 \mu \mathrm{M}$ dGTP, $20 \mu \mathrm{Ci} \alpha-{ }^{32} \mathrm{P}$-dGTP, $10 \mathrm{mM}$ DTT, 5' biotinylated DNA substrate (5'-CTAGACCTGTCATCA(TTAGGG) ${ }_{3}-3^{\prime}$ ) and $10 \%$ glycerol. The reaction was conducted at $37^{\circ} \mathrm{C}$ for $2 \mathrm{~h}$. The purification of telomerase extension products employed Dynabeads M-280 streptavidin. Five microliters of purified products were loaded on $6 \%$ sequencing gel (TBE-UREA denaturing gel) with Model S2 Sequencing Gel Electrophoresis Apparatus (LabRepCo, Horsham, PA). The gel was dried at $80^{\circ} \mathrm{C}$ for $30 \mathrm{~min}$ and exposed to phosphorimaging screen for $2 \mathrm{~h}$. This screen was then scanned using a STORM phosphorimager (Molecular Dynamics), and bands were quantified using GelAnalyzer as described above.

The $\mathrm{EC}_{50}$ was the extracellular concentration of MPP inhibitor calculated to result in $50 \%$ reduction of cellular telomerase activity after incubation in whole cells. Similarly, $\mathrm{IC}_{50}$ was the concentration of MPP inhibitor necessary to inhibit 50\% of telomerase enzyme activity in vitro in cellular lysates. Both were calculated using GraphPad as directed and verified graphically on plots of enzyme activity vs inhibitor concentration.

\section{8 | Non-denaturing agarose gel electrophoresis}

0.8\% Agarose gels were run in Tris-Borate-EDTA buffer using standard slab gels as described for high molecular weight complexes. ${ }^{27}$
Cell lysates were produced by lysing Hek293 and Huh7 cells in NP40 buffer (25 mM HEPES-KOH, $150 \mathrm{mM} \mathrm{KCl}, 1.5 \mathrm{mM} \mathrm{MgCl}$, $10 \%$ glycerol, 0.5\% NP40, 5 mM 2ME, pH 7.5 supplemented with protease inhibitors) for $30 \mathrm{~min}$ on ice. Extracts were clarified by centrifugation for $16000 \mathrm{~g}$ for $10 \mathrm{~min}$. The protein concentration was determined by Bradford assay. The indicated amounts of proteins were treated with different amounts of ZnPP for $2 \mathrm{~h}$ on ice, and then separated on $0.8 \%$ Agarose gels prepared with $0.5 x$ Tris-Borate-EDTA (TBE) buffer. The gels were run at $100 \mathrm{~V}$ for $2 \mathrm{~h}$ at $4^{\circ} \mathrm{C}$ in $0.5 \mathrm{x}$ TBE buffer. To further demonstrate the binding of ZnPP to telomerase complex, immunoprecipitation was performed using 300 $\mathrm{g}$ of Hek293 lysate with hTERT antibody (Y182, Abcam). No antibody and normal rabbit IgG (sc-2027, Santa Cruz Biotechnology) were included as controls. The IP products were treated with ZnPP and electrophoresed as above. Free ZnPP and ZnPP bound to large complexes were visualized using a wide-wavelength UV lightbox or with red fluorescence using excitation (EX) 608-632 nm and emission (EM) 675-720 nm. Thyroglobulin (670 kD), a heavy MW marker, was run in parallel lanes and visualized with Coomassie Blue staining or fluorescence to size the complexes. In some cases, cell lysates or IP were incubated with RNase A (Life Technologies, NY); or DNase 1 (Qiagen, CA) $100 \mu \mathrm{g} / \mathrm{ml}$ for $1 \mathrm{~h}$ at $4^{\circ} \mathrm{C}$ prior to electrophoresis.

Diffusion blotting of $0.8 \%$ agarose native gels: After electrophoresis, protein complexes were transferred to nitrocellulose (NC) membranes using direct capillary action overnight at RT in the presence of $1 x$ Tris-buffered saline (TBS). Then the NC membranes were washed with fresh TBS and immunodetection of complexes performed as described below for western blot assays.

\section{9 | TERT overexpression and Immunoprecipitation}

Plasmid pCl neo-hEST2 together with TERC (pBS U3-hTR-500) were transfected into log phase Hek293 cells and non-denaturing cellular lysates were prepared $48 \mathrm{~h}$ later in lysis buffer (Cell Signaling Technology, Beverly, MA). Immunoprecipitation was performed as described previously. ${ }^{21}$ Briefly, transfected cells were harvested, washed in PBS, lysed in cell lysis buffer, and clarified by cold centrifugation (14 $000 \times \mathrm{g}$ for $10 \mathrm{~min}$ ). An aliquot of supernatant containing $500 \mu \mathrm{g}$ protein was incubated with $2 \mu \mathrm{g}$ anti-hTERT antibody MABE14 (EMD Millipore, MA) at $4^{\circ} \mathrm{C}$ overnight with gentle mixing. Then, $20 \mu$ l of recombinant Protein G Agarose (Invitrogen, CA) was added and incubated at $4^{\circ} \mathrm{C}$ for $3 \mathrm{~h}$. IPs were collected by centrifugation at 3,000 rpm for $30 \mathrm{~s}$ at $4^{\circ} \mathrm{C}$, washed three times with ice-cold PBS, aliquoted, and stored at $-80^{\circ} \mathrm{C}$ until use. For denaturing gel electrophoresis, aliquots were dissolved in $2 \mathrm{X}$ Laemmli electrophoresis sample buffer (Bio-Rad, CA) and assayed by western blot (WB). Normal rabbit or mouse IgG was always used as a control (Santa Cruz, CA). Aliquots of the IP were also assayed in triplicate by TRAP assay and quantified using realtime PCR as described above. In some cases, aliquots were electrophoresed on non-denaturing agarose gels after treatment with MPP and/or nucleases. 


\subsection{0 | SDS-Polyacrylamide gel electrophoresis (PAGE) and Western blot assays}

For SDS -PAGE, cellular lysates, and protein preparations such as IP were dissolved in Laemmli buffer, boiled for 1 min and separated on denaturing SDS gels as described. ${ }^{28}$ After electrophoretic transfer of separated proteins to nitrocellulose sheets, western blot immunoassays employed enhanced chemiluminescence for signal detection $\left(E C L^{T M}\right.$ Prime, Amersham). ${ }^{28}$

\subsection{1 | Cellular fluorescence labeling}

Cells were grown to semi-confluency while attached to coverslips, then washed in PBS, fixed in absolute methanol, re-washed in PBS, then incubated with anti-TERT antibodies with or without $10 \mu \mathrm{M} \mathrm{ZnPP}$ for $1 \mathrm{~h}$ at RT. Cells were washed in PBS, then incubated with secondary antibodies conjugated to the fluorochromes Alexa Fluor 488 (green). Slides were mounted with VECTASHIELD H-1000 (Vector Labs, Burlington, Ontario) and counterstained with To-pro $^{\mathrm{TM}}-3$ lodide (ThermoFisher Scientific) to visualize nuclei. Confocal microscopy was performed on a Zeiss LSM710 confocal fluorescence microscope. Alexa Fluor 488 (green) and Alexa Fluor 568 (red) filters were used to visualize TERT and ZnPP respectively.

\subsection{2 | Preparation of metaphase chromosomes}

Huh7 cells were cultured in regular DMEM with colcemid $(0.1 \mu \mathrm{g} / \mathrm{ml})$ for $2 \mathrm{~h}$ at $37^{\circ} \mathrm{C}$. Cells were harvested, pelleted by centrifugation, supernatant removed, then re-suspended in a solution of warm $\left(37^{\circ} \mathrm{C}\right)$ $0.075 \mathrm{M} \mathrm{KCl}$, and incubated for $20 \mathrm{~min}$ in a $37^{\circ} \mathrm{C}$ water bath. The cells were pre-fixed by adding fixative (3:1 ethanol/acetic acid) and centrifuged for $5 \mathrm{~min}$ at 1000 RPM at room temperature. The supernatant was removed, and the cells suspended in the fixative solution. The metaphase chromosomes were then spread on slides and reacted with antibodies for immunofluorescence or labeled with ZnPP or telomere probe as described above.

\subsection{3 | S-phase synchronized cells}

To synchronize cells in S phase, we performed double thymidine block essentially as described ${ }^{29}$ with modifications. Briefly, Huh7 cells were seeded onto coverslips and then treated with $2 \mathrm{mM}$ thymidine (Sigma, T9250) for $18 \mathrm{~h}$, released for $9 \mathrm{~h}$, again treated with $2 \mathrm{mM}$ thymidine for $18 \mathrm{~h}$, then released $2 \mathrm{~h}$ before use. To determine the optimal timepoint for collecting cells in $\mathrm{S}$ phase, we assayed cultures with flow cytometry and Western blot for cyclin A expression (Figure S4). The cells were fixed with $4 \%$ paraformaldehyde and washed with PBS. Telomere FISH and ZnPP staining was then conducted as described below.

\subsection{Fluorescence in situ hybridization (FISH)}

We performed telomere $\mathrm{FISH}^{16}$ using a peptide nucleic acid (PNA) probe specific to telomeres, and labeled with TelC-Alexa488 (PNA Bio, Newbury Park, CA,) as per the manufacturer's instructions with modifications. Briefly, $0.2 \mu \mathrm{l}$ of PNA probe was added to $20 \mu \mathrm{l}$ of hybridization solution which was used to cover cells attached to slides. Hybridization was at $80^{\circ} \mathrm{C}$ for $10 \mathrm{~min}$, then $37^{\circ} \mathrm{C}$ overnight. After washing, slides were incubated with $10 \mu \mathrm{M} \mathrm{ZnPP} 2 \mathrm{~h}$ at room temperature, washed twice in PBS, and mounted with VECTASHIELD. Confocal fluorescence microscopy used a Zeiss LSM710 confocal fluorescence microscope using $63 x$ oil objective.

\subsection{Statistical determinations}

All mean values for enzymatic and proliferation assays were determined using 3-6 replicates per point. Data are plotted as the mean value of each point \pm standard deviation. For all figures, when $a \pm$ bar is not seen, the SD was smaller than the graph symbol. Graphpad Prism or Excel software was used for least squares regression, calculation of all variances, and curve placement. A completely randomized design with multiple treatment groups was used for the analysis of variance (ANOVA) for each experiment and variances then pooled among experiments using appropriate degrees of freedom for among and within group comparisons. $\mathrm{IC}_{50}$ and $\mathrm{EC}_{50}$ values (as defined above) were determined by regression assuming sigmoid inhibition curves using GraphPad Prism software and tested pairwise using t-statistic assuming normal distribution. All experiments were repeated at least twice, and the authenticity of the findings verified on three or more occasions.

\subsection{Nomenclature of targets and ligands}

Key protein targets and ligands in this article are hyperlinked to corresponding entries in http://www.guidetopharmacology. org, the common portal for data from the IUPHAR/BPS guide to PHARMACOLOGY ${ }^{30}$ and are permanently archived in the Concise Guide to PHARMACOLOGY 2019/2031

\section{3 | RESULTS}

We first compared the effects of various MPPs (FePP, ZnPP, SnPP and CoPP) on cellular proliferation and DNA synthesis, (Figure 1A-C) in Huh7 hepatoma and Hek293 embryonic kidney cells known to express telomerase. In contrast to other MPPs, ZnPP severely attenuated DNA synthesis (Figure 1A) and depressed cellular proliferation greater than $50 \%$ at $48 \mathrm{~h}$ in both cell lines relative to vehicle only controls (Figure 1B-C).

Further experiments documented that ZnPP had only minor effects on proliferation in telomerase negative U2OS cells, (Figure 1D, 
(A) ZnPP inhibits thymidine incorporation
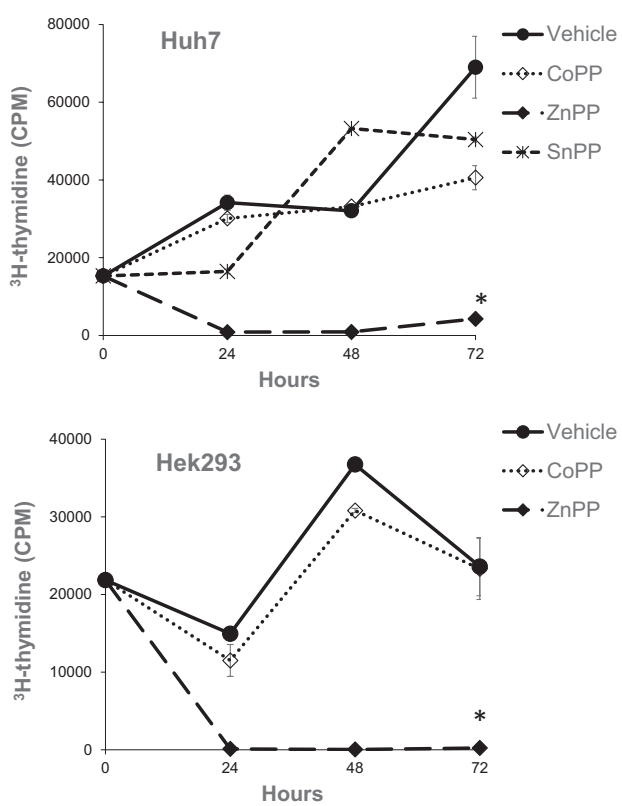

(B) Effects of MPPs on cellular proliferation
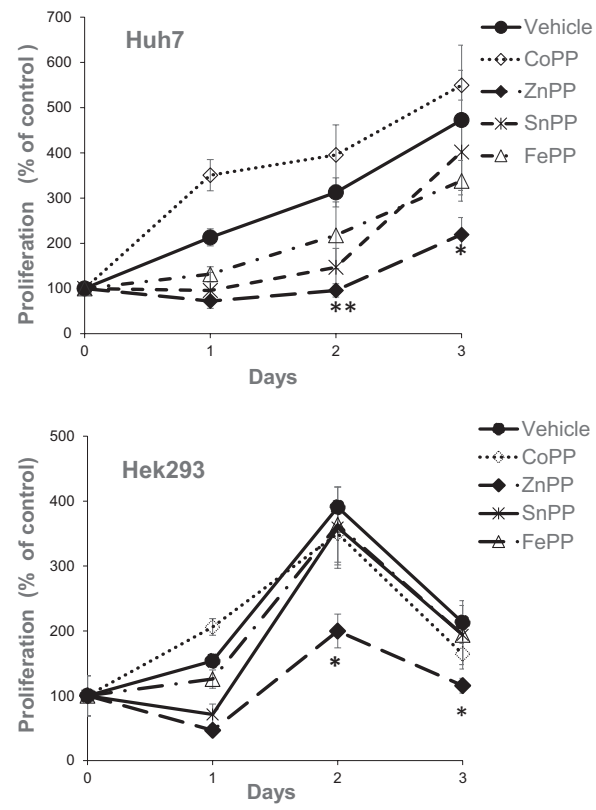

FIGURE 1 Effects of MPPs on DNA synthesis, cellular proliferation, and viability. (A) Incorporation of ${ }^{3} \mathrm{H}$-thymidine. Early log phase cultures of Huh7 (upper chart) or Hek293 (lower chart) cells were treated with $10 \mu \mathrm{M}$ of the indicated MPP. The incorporation of ${ }^{3} \mathrm{H}$ thymidine was determined at 24,48 , or $72 \mathrm{~h}$ of MPP treatment. $t$-test, ZnPP versus other MPPs, ${ }^{*} p<.001$. (B) Inhibition of proliferation. MTT assays were performed after $10 \mu \mathrm{M}$ MPP treatment of Huh7 cells (upper chart) or Hek293 cells (lower chart) for 0, 1, 2, 3 days. $t$-test, ZnPP versus other MPPs, ${ }^{*} p<.01,{ }^{* *} p<.05$. (C) Cell growth and viability. ZnPP (upper chart) and FePP (lower chart) were added to log phase cultures of Huh7 cells. The number of viable cells that excluded trypan blue dye was determined daily using direct cell counting. $t$-test 5 and $10 \mu \mathrm{M}\left({ }^{*} p<.05\right)$, and $20 \mu \mathrm{M}^{* *} p<.01$ versus other concentrations at days 2 and 3, respectively. (D) Effects of ZnPP in TERT negative (U2OS) as compared to TERT positive (Huh7) cell lines. Early log phase cells were treated with the indicated concentrations of ZnPP for $48 \mathrm{~h}$ (upper chart) or BIBR for $72 \mathrm{~h}$ (middle chart) and viability then determined using MTT assay. Upper chart, $t$-test U2OS versus Huh7 ${ }^{*} p<.01$. Middle chart, $t$-test, U2OS versus Huh7 at 10 and $20 \mu \mathrm{M}{ }^{*} p<.01$. For lower chart, vector only plasmid or plasmid pCI neo-hEST2 containing complete human TERT DNA sequences were transfected into log phase U2OS cells. $24 \mathrm{~h}$ later, cultures received the indicated concentrations of ZnPP and then incubated $48 \mathrm{~h}$, followed by MTT assay to determine viability. $t$-test, TERT vs vector control cells viability ${ }^{*} p<.01$. (Right) Immunoblot analysis of TERT in U2OS cells after sham treatment or transfections with plasmid only or human TERT sequences. Untreated Huh7 cell lysate with endogenous TERT is shown as positive control. (E) Apoptosis was measured with BD Biosciences Annexin V FITC assay in Hek293 (upper), Huh7 (middle) and U2OS (lower) panels cells after incubation with the indicated concentration of MPP for 48hr. ANOVA with paired t- test, ZnPP apoptosis versus other MPPs, ${ }^{* *} p<.025,{ }^{*} p<.01$. (A-E) Variability expressed as mean, $+/$ standard deviation, $n=3-6$ determinations per point

upper chart). As a positive control, BIBR1532, a known mixed-type non-competitive inhibitor of telomerase ${ }^{32}$ was tested in the same cell lines. BIBR also showed greater propensity to decrease proliferation in telomerase-expressing rather than a telomerase negative line in accordance with earlier reports ${ }^{33}$ (Figure 1D, middle chart). In contrast, transfection of TERT sequences into U2OS restored ZnPP sensitivity that was quite similar to that of Huh7 cells (Figure 1D, upper and middle charts respectively). The immunoblot of Figure 1D (lower panel) documented that TERT transfected U2OS cells avidly expressed TERT as compared to vector-only controls.

Consistent with effects on DNA synthesis and proliferation, ZnPP was the most effective MPP at inducing apoptosis (ca.: $50 \%$ at $10 \mu \mathrm{M}$ ) in TERT positive Huh7 or Hek293 cells in contrast to other MPPs evaluated (Figure 1E, upper and middle charts respectively). On the other hand, ZnPP failed to have an increased effect on apoptosis in telomerase negative U2OS cells (Figure 1E lower chart). Taken together, these findings suggest that ZnPP interactions with the telomerase system contribute to the effects on apoptosis and anti-proliferative behavior.

Because of ZnPP actions on DNA synthesis, proliferation, and apoptosis in TERT positive cells, we determined ZnPP effects on TERT expression with western blots (WB) (Figure 2A). As positive controls, we also evaluated other pro-proliferative proteins, $\beta$-catenin and cyclin D1 which have been closely linked to TERT expression and signaling. ${ }^{34,35} \mathrm{ZnPP}$ reduced expression of all three proteins by 8-24 $\mathrm{h}$ and by $48 \mathrm{~h}$ expression was nearly eliminated (Figure $2 \mathrm{~A}$ ). Cells treated with various concentrations of $\mathrm{ZnPP}$ for $48 \mathrm{~h}$ (Figure 2A, left middle panel) further confirmed these findings. In contrast, CoPP, FePP, or SnPP failed to significantly alter TERT, $\beta$-catenin, or cyclin D1 expression (Figure 2A, right middle and bottom panels) consistent 
(C) Effects of MPPs on cell growth and viability

(D) ZnPP action in TERT negative cells
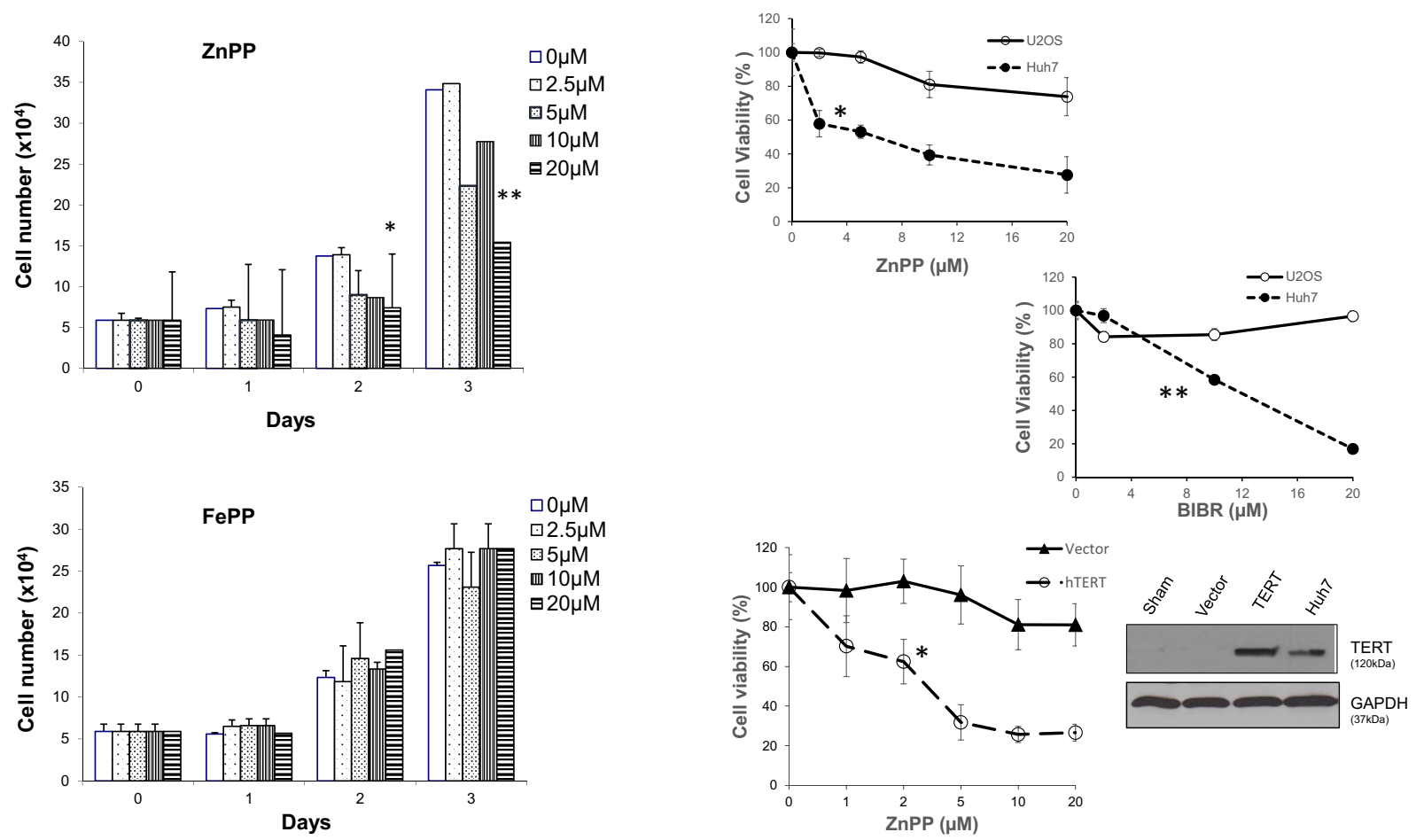

(E) Effects of various MPPs on apoptosis

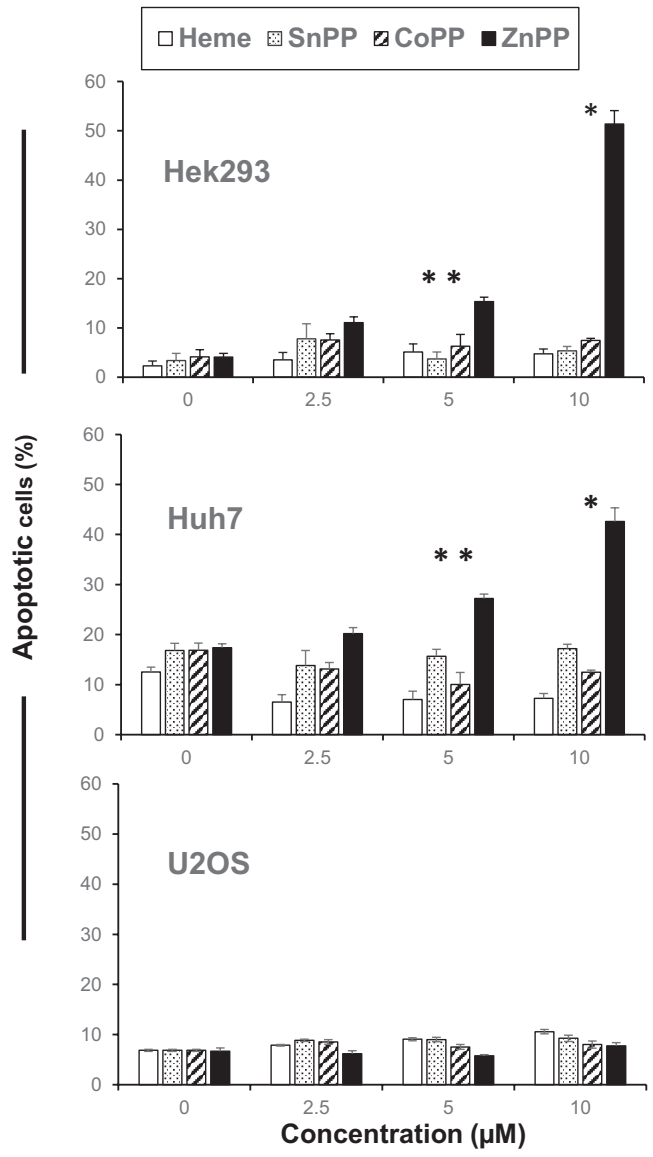

FIGURE 1 (Continued) 
with their minimal effects on DNA synthesis and apoptosis (Figure 1). The effects of ZnPP on TERT expression were apparent in different Huh 7 constructs and, interestingly, in a NS 5.15 HCV replicon, ZnPP promoted disappearance of both $120 \mathrm{kDa}$ telomerase monomer as well as the $45 \mathrm{kDa} C$-terminal TERT fragment that we previously reported to be specific for HCV infected cells (Figure 2B). ${ }^{21}$

We next evaluated the effects of MPPs on telomerase activity in cultured cells (Figure $3 \mathrm{~A}$ ) as well as non-denatured cell lysates (Figure 3B-E). In cultured cells incubated with ZnPP, telomerase activity was reduced in a dose-dependent fashion, $\left(E_{50}=5.6-5.8 \mu \mathrm{M}\right.$, upper and middle panels respectively) while SnPP or FePP, had none to mild effects ( $\mathrm{EC}_{50}>10 \mu \mathrm{M}$, either cell line) (Figure $3 \mathrm{~A}$ ). The loss of telomerase activity with time of ZnPP treatment in the NS 5.15 $\mathrm{HCV}$ replicon (Figure $3 \mathrm{~A}$, lower panel) reflected the disappearance of TERT seen in the WB (Figure $2 B$ ).

The possibility that MPPs can directly inhibit telomerase activity in cellular extracts, similar to porphyrin quadruplex ligands, ${ }^{6}$ was addressed next. Because of concerns that some quadruplex ligands inhibit Taq DNA polymerase in addition to telomerase, we assayed MPP inhibition at both steps of the TRAP procedure with a strategy similar to that of others. ${ }^{36}$ Using equivalent extracts but separate assays, MPP was either included in the telomerase RT extension step or the extension step was conducted without MPP and then MPP added only for the amplification steps with Taq DNA polymerase. To avoid further potential errors introduced by Real-time quantification, TRAP products were labeled with SYBR green, visualized on denaturing gels, and each lane was quantified by density measurements as described in the Methods. The latter step also ruled out the possibility that decreases in activity were artifactual due to fluorescence signal quenching by some MPPs. ${ }^{37} \mathrm{ZnPP}$ was significantly more active $\left(\mathrm{IC}_{50}=2.5 \mu \mathrm{M}\right.$ ) than FePP and SnPP (both $I_{50}>10.0 \mu \mathrm{M}$ ), (Figure 3B-D respectively). All three MPPs had minimal effects on Taq polymerase during telomerase product extension and the slight inhibition of Taq polymerase seen for ZnPP was not directly concentration-dependent. However, at least one MPP, CoPP, clearly inhibited Taq polymerase and could not be reliably assayed via TRAP assay (see Figure S2).

To confirm that ZnPP specifically inhibited telomerase as seen in the TRAP assays, direct telomere extension assays were conducted in the presence of $\alpha^{-}{ }^{32} \mathrm{P}-\mathrm{dGTP}$. An IC $\mathrm{IC}_{50}$ of $3.8 \mu \mathrm{M}$ for ZnPP obtained by direct extension assay (Figure $3 \mathrm{E}$ ) was quite similar to the $\mathrm{IC}_{50}$ obtained with TRAP assay $(2.5 \mu \mathrm{M})$ (Figure 3B). Consequently, by two independent assay procedures, ZnPP was observed to directly inhibit telomerase activity in cellular extracts and the $I_{50}$ values are roughly within a twofold range of the $\mathrm{EC}_{50}$ values for intact Huh7 and Hek293 cells ( 5 and $6 \mu \mathrm{M}$ respectively). $\mathrm{IC}_{50}$ and $\mathrm{EC}_{50}$ values of the three MPPs obtained by different assay procedures are summarized in Table 2.

ZnPP, SnPP, and non-metal, "free" Lewis base protoporphyrins exhibit autofluorescence ${ }^{37}$; a property that has proven useful to study intracellular activities of MPPs such as nuclear localization and DNA or cellular adduct binding. ${ }^{13,23,38,39}$ Other transition

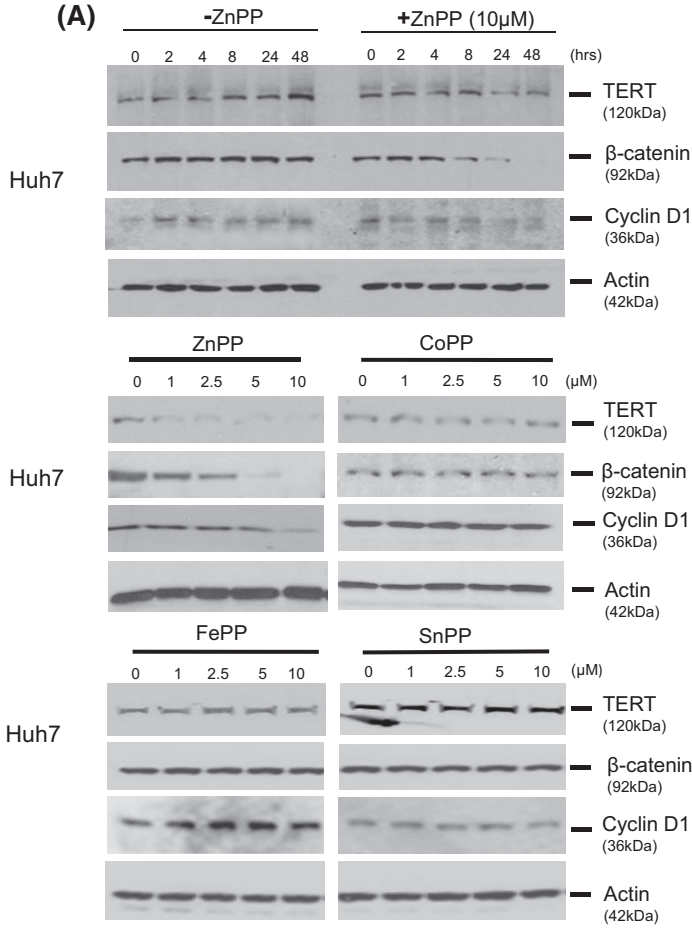

(B)

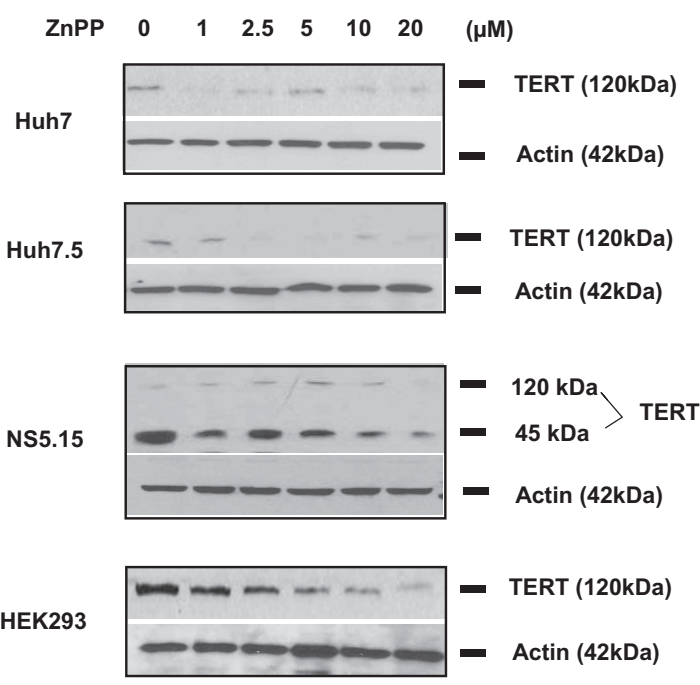

FIGURE 2 MPP effects on cellular expression of proliferative proteins TERT, $\beta$-catenin, and cyclin D1. (A) ZnPP decreases expression of proliferative proteins. Log phase Huh7 cells were treated with the indicated concentrations of MPPs and assayed for various times, (upper panel) or after $48 \mathrm{~h}$ (middle and lower panels) on WB using specific antibodies. (B) Log phase cultures of different clonal Huh7 and Hek293 cell lines were incubated with the indicated concentrations of ZnPP for $48 \mathrm{~h}$. TERT was then identified on WB with specific anti-TERT antibodies. [ ${ }^{*} \mathrm{HCV}$ positive replicon $\left.{ }^{21}\right]$ 
(A) Telomerase inhibition by MPP in intact cells

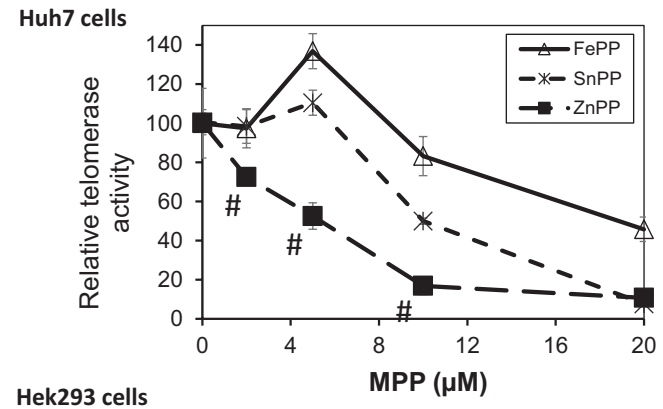

Hek293 cells

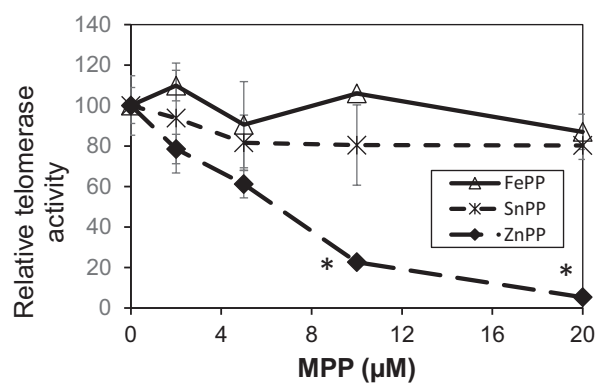

NS 5.15 HCV Replicons

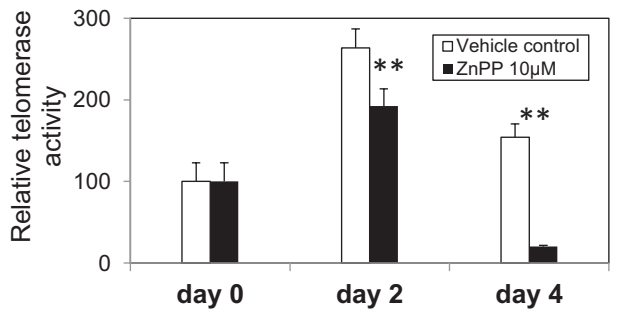

(B) ZnPP Inhibition of telomerase activity in telomeric extension assays
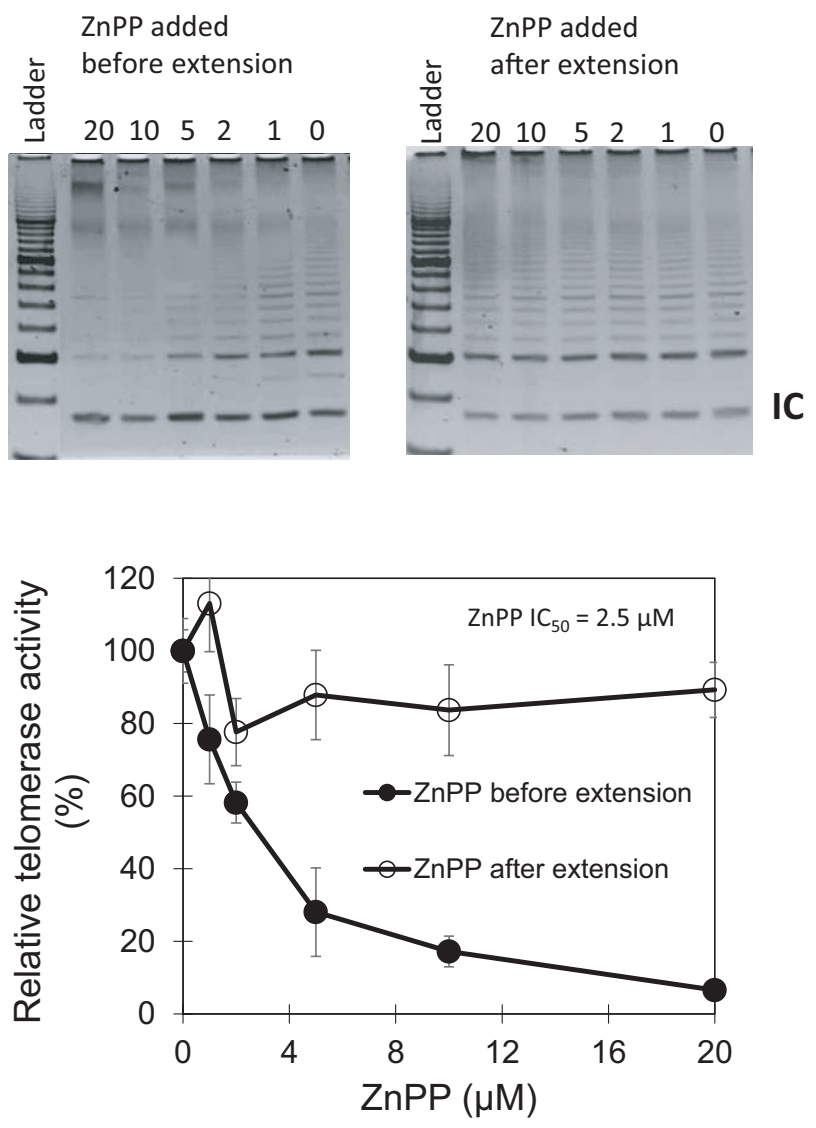

FIGURE 3 MPP inhibition of telomerase enzymatic activity. (A) Telomerase inhibition by MPP in cultured cells. Log phase Huh7, Hek293 and Huh5.15 HCV replicon cells, (upper, middle, and lower panels, respectively), were incubated with MPPs (ZnPP, SnPP, or FePP) for 48 $\mathrm{h}$, then telomerase activity was determined by TRAP assay in enzymatic lysate. Points represent mean $\pm \mathrm{SD}, n=6, A N O V A$, \#p $<.01$, ${ }^{*} p<.001,{ }^{* *} p<.01$ paired t-test of ZnPP versus other MPPs or vehicle only controls. (B-D) Telomerase inhibition by MPP in enzymatic extracts. Enzymatic extracts were prepared from semi-confluent phase Huh7 cells and aliquots were assayed in triplicate using TRAP assay. MPPs were added to RT-PCR reactions either before or after the RT telomere elongation step to test whether MPPs inhibit Taq polymerase. Visualization of amplified telomeric products was on $10 \%$ non-denaturing polyacrylamide gels using SYBR fluorescence labeling (upper panels) followed by quantification with densitometry and plotted (lower panel). IC = Internal Taq polymerase control. Plotted points represent the mean \pm SD with $n=3$. (E) MPP inhibition of telomerase activity as determined with direct $\alpha{ }^{-32}$ P-dGTP extension assays. Aliquots of IP eluates from $10^{7} \mathrm{Hek} 293$ cell pellet lysates overexpressing hTERT, TERC, and dyskerin were incorporated into direct telomere extension assays using biotin-linked DNA substrate. Reactions were incubated at $37^{\circ} \mathrm{C}$ for two hours, then biotin labeled products purified with strepavidin linked agarose beads and electrophoresed on denaturing acrylamide gels. Bands were visualized radiographically (upper panel) and relative activity was quantified with densitometry (lower panel). Points represent mean $\pm \mathrm{SD}, n=3, \mathrm{ANOVA} * p<.01$ paired test of ZnPP versus other MPPs

metal MPPs such as FePP or CoPP are inactive fluoroscopically because they have unfilled transition metal $d$ orbitals that quench fluorescence emission. We investigated ZnPP binding to native, nondenatured telomerase-containing complexes after separation on large pore, (0.8\%), agarose gels. ${ }^{27,40}$ Initially, non-denaturing acrylamide gels were considered for these studies, however, we discovered that ZnPP labeled complexes would not enter the largest pore size possible, a result also noted by others ${ }^{23,39}$ (see Figure S3).

Initially, increasing amounts of cellular extracts were incubated with varying amounts of $\mathrm{ZnPP}$, then electrophoresed on large pore agarose gels. ZnPP was then visualized fluoroscopically using visible red wavelengths [608-632 nm Ex and 675-720 nm EM] or wide band UV (Figure 4A upper and lower panels respectively). ZnPP bound to high molecular weight complexes in a concentration dependent manner and the complexes electrophoresed with a mobility just above thyroglobulin ( $670 \mathrm{kD}$ ), quite similar to sizes noted by us and others for TERT ribonuclear protein particles separated by glycerol gradient centrifugation ${ }^{21,41}$ and large pore agarose/acrylamide gels. ${ }^{40}$ Under these conditions, free ZnPP migrated slightly cathodal. While ZnPP binding was easily identified in cellular extracts, no binding was detectable in bulk protein incubations of BSA or IgG (Figure 4B). ZnPP also labeled complexes in intact cells 
(C) FePP inhibition of telomerase activity in telomeric extension assays
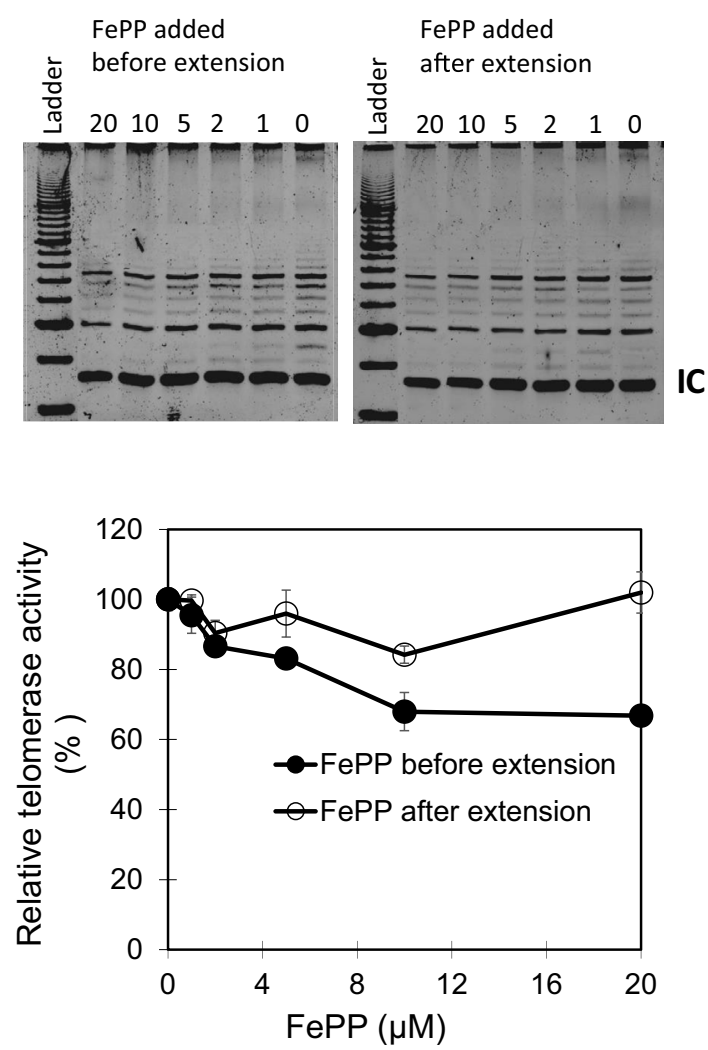

FI GURE 3 (Continued)

as determined by electrophoresis of extracts prepared after ZnPP incubation in culture (Figure 4C).

To assess whether TERT is a component of the ZnPP-labeled cellular extracts we incubated lysates with anti-human TERT, $\beta$ catenin, or cyclin D1 antibodies or non-specific IgG antibodies prior to electrophoresis and looked for upward mobility shift after electrophoresis (Figure 4D). Only anti-TERT antibody led to a significant upward mobility shift of ZnPP labeled complexes from either cell type, suggesting that TERT is indeed a component of the large complexes. No mobility shift was noted for the other antibodies tested suggesting ZnPP specifically labeled TERT complexes. Note that both cyclin D1 and $\beta$-catenin would be expected to be components of large molecular complexes in non-denatured cellular lysates. ${ }^{42,43}$ Further characterization of the ZnPP binding complexes as to protein and DNA composition, and investigation of SnPP binding is presented in the online Supplemental Data (Figure S3).

ZnPP labeled complexes from Huh7 cells were next blotted onto nitrocellulose by capillary diffusion (conditions determined empirically, see Figure S4) and probed with specific anti-TERT or anti-dyskerin antibodies, the latter a positive control for telomerase holoenzyme (Figure 4E). Both TERT and dyskerin were easily identified in the high molecular weight complexes binding ZnPP (Figure 4E). Interestingly, cellular lysates showed more immunoreactive TERT
(D) SnPP Inhibition of telomerase activity in telomeric extension assays
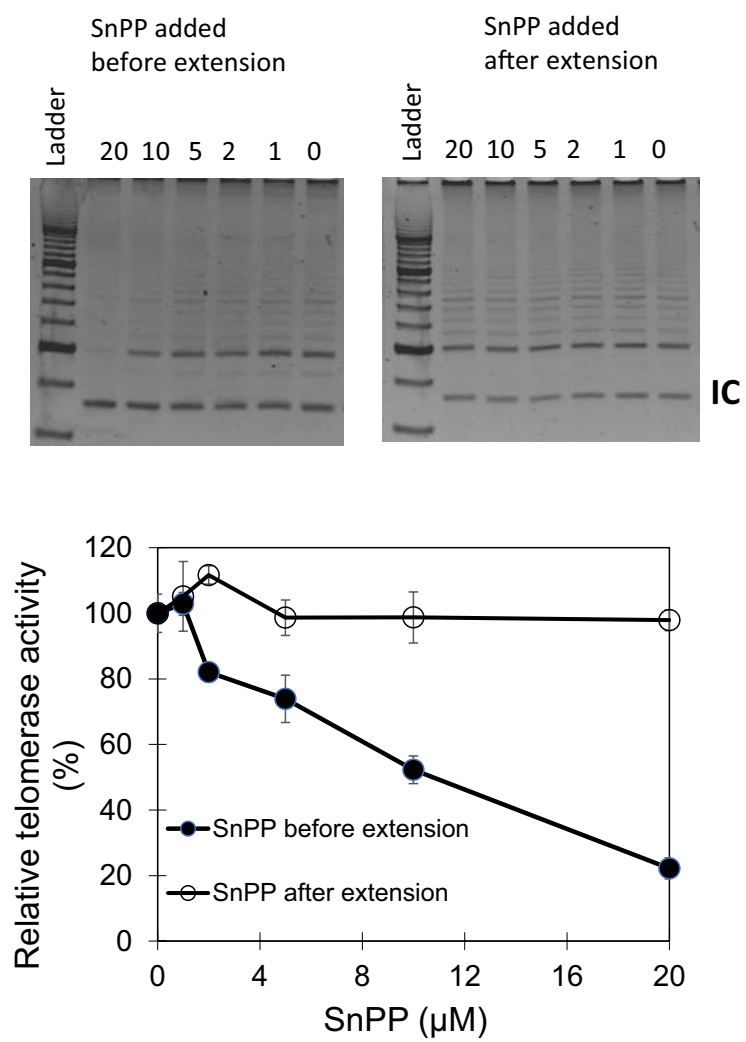

when incubated with ZnPP prior to electrophoresis suggesting a protective effect of ZnPP on TERT in the extracts.

Immunoprecipitation using TERT-specific antibodies further confirmed that TERT is a component of ZnPP labeled complexes. Immunoprecipitates (IP) were evaluated on native agarose as well as denaturing SDS gels and WB (Figure 5A, left and right panels respectively). ZnPP bound the anti-TERT IP complexes intensely and IP had increased mobility as compared to non-specific IP complexes or no antibody control (Figure 5A, left panel). As expected, the antiTERT IP analyzed on WB (Figure 5A right panel) showed increased TERT as compared to IP from non-specific or no antibody controls. A weaker TERT band (relative to exposure time) was also confirmed in the crude cellular lysate. When assayed by TRAP assay, IP TERT complexes and unpurified enzyme showed similar $\mathrm{IC}_{50}$ values with ZnPP, (3.1 vs. $2.2 \mu \mathrm{M}$, respectively), (Figure 5B). These measurements were also close to the $\mathrm{IC}_{50}$ observed for overexpressed enzyme, $(3.8 \mu \mathrm{M})$, when assayed by direct $\alpha-\mathrm{P}^{32}$-dGTP extension assay (Figure 3E), (Table 2).

To investigate whether nucleic acids are components of the ZnPP labeled complexes, IP or crude lysates, were digested with DNase I or RNase A prior to labeling with ZnPP (Figure 5C, upper panel). Digestion of extracts with DNase I elicited minimal changes in the mobility of ZnPP labeled complexes, however, a significant upward shift was seen when extracts were digested with RNase 
(E) MPP inhibition of telomerase activity by direct $\alpha-{ }^{32} P$ -

dGTP extension assay
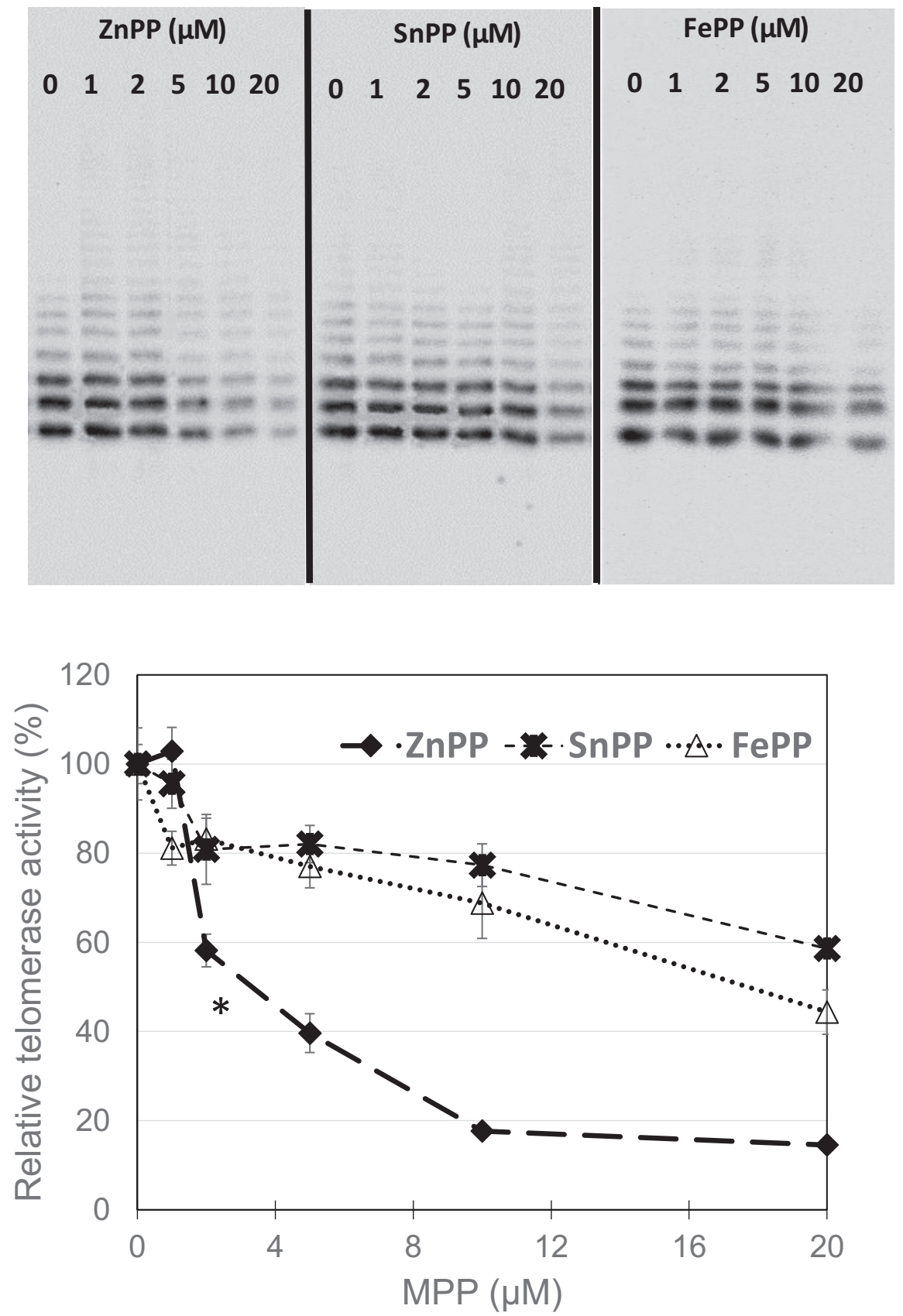

FIGURE 3 (Continued)

A. Furthermore, there was a marked increase in unbound ZnPP after nuclease, most dramatic with RNase A-treated IPs (arrow, Figure 5C, upper panel) suggesting that ZnPP most likely binds to a ribonuclear protein complex and the binding site is at least partially disrupted with RNase digestion. Next, nuclease digested, ZnPP labeled complexes were blotted onto nitrocellulose and reacted with anti-TERT antibodies. These experiments showed that RNase A digestion severely diminished the amount of immunoreactive TERT in the labeled complexes, most notably for IP complexes (Figure 5C, lower panel). These findings strengthened the conclusion that ZnPP binds to high molecular weight telomerase complexes. In addition to providing a telomere template, TERC is an important structural component of telomerase holoenzyme and RNase A digestion is known to completely disrupt ribonuclear complex structure and release TERT. $^{44}$

ZnPP binding to cellular structures in situ was probed by confocal immunofluorescence microscopy. While telomere sequences exist throughout the mammalian chromosome, it is known that 


\begin{tabular}{|c|c|c|c|c|}
\hline MPP & $I C_{50}(\mu \mathrm{M})$ & ME & $\mathrm{R}^{2}$ & $\begin{array}{l}\text { P values of } I_{50} \\
{[Z n P P<S n P P \text { or }} \\
\text { FePP] }\end{array}$ \\
\hline
\end{tabular}

TABLE 2 Inhibition of telomerase by MPPs in direct telomere extension assays

a. Cellular lysates [Trapeze]

$\begin{array}{lllll}\text { ZnPP } & 2.5 & 4.39 & 0.974 & \\ \text { SnPP } & 12 & 2.35 & 0.962 & p<.001 \\ \text { FePP } & >20 & 7.9 & 0.911 & p<.001\end{array}$

b. $\alpha{ }^{-32} \mathrm{P}-\mathrm{dGTP}$ direct telomerase

assay

ZnPP

3.8

1.64

0.904

SnPP

$>20$

2.32

0.645

FePP

18.3

2.75

0.777

$p<.01$

c. Intact Huh7 cells [TRAP]

\begin{tabular}{|c|c|c|c|c|}
\hline & $\mathrm{EC}_{50}(\mu \mathrm{M})$ & & & \\
\hline ZnPP & 5.4 & 2.89 & 0.941 & \\
\hline SnPP & 10.8 & 4.76 & 0.865 & $p<.01$ \\
\hline FePP & $>20$ & 9.6 & 0.405 & $p<.01$ \\
\hline \multicolumn{5}{|c|}{ d. Intact Hek293 cells [TRAP] } \\
\hline $\mathrm{ZnPP}$ & 6.4 & 2.41 & 0.928 & \\
\hline $\mathrm{SnPP}$ & $>20$ & NC & 0.076 & $p<.001$ \\
\hline FePP & $>20$ & NC & 0.085 & $p<.001$ \\
\hline \multicolumn{5}{|c|}{$\begin{array}{l}\text { e. TERT IP vs cellular lysate } \\
\text { [TRAP] }\end{array}$} \\
\hline & $\mathrm{IC}_{50}(\mu \mathrm{M})$ & & & $I_{50}$ of IP vs lysate \\
\hline ZnPP (IP) & 2.4 & 4.8 & 0.953 & NS \\
\hline ZnPP (lysate) & 1.8 & & 0.963 & \\
\hline
\end{tabular}

Note: ZnPP, SnPP and FePP telomerase inhibition in intact cells, cellular lysates and anti-TERT immunoprecipitates. $\mathrm{R}^{2}$ values reflect regression analysis assuming sigmoidal inhibition curve. IC ${ }_{50}$ and $\mathrm{EC}_{50}$ values were determined as directed by GraphPad software. $I C_{50}$ and $\mathrm{EC}_{50}$ values are presented as +/- the margin of error (ME) determined at $99 \%$ confidence level or greater assuming $\mathrm{t}$ distribution. Each $\mathrm{IC}_{50}$ or $\mathrm{EC}_{50}$ reflects mean values of 2-4 experiments using 6 concentrations of inhibitor per curve $(0,1,2.5,5,10$, and $20 \mu \mathrm{M})$ and 3-6 determinations per point. Mean values may differ slightly from values reported in the text for individual experiments.

telomerase holoenzyme only associates with telomeres at DNA replication during S phase. ${ }^{45}$ Consequently, we compared ZnPP localization in synchronized S-phase cells as compared to metaphase chromosomes which contain prominent telomere ends without telomerase. The percentages of cells in S phase were determined temporally with flow cytometry after double thymidine block and extracts were monitored on immunoblots with Cyclin A2 staining to determine optimal times for study, (Figure S5). Telomere sites were labeled with telomere sequence-specific fluorescent probe (PNA TelC-Alexa488) and TERT was localized with specific antibodies (Figure 6).

First, we looked at ZnPP co-localization with the telomere probe. While ZnPP clearly localized with telomeres in $\mathrm{S}$ phase cells (Figure 6A left panels), it did not label the prominent telomeres on metaphase chromosome tips, (Figure 6A, right panels) which are devoid of holoenzyme. Next, we investigated whether ZnPP would co-localize with TERT in S phase as compared to unsynchronized Huh7 cells. In S phase cells, TERT co-localized with
ZnPP in the nucleus and at some cytoplasmic sites (Figure 6B left panels). As we reported previously, TERT is sparsely present in the nucleus of unsynchronized, log phase Huh7 cells, but is chiefly found at perinuclear sites which co-localize with mitochondria. ${ }^{21}$ Interestingly, even perinuclear TERT, likely lacking telomeric DNA, showed avid TERT- ZnPP co-localization (Figure 6B, right panels). Collectively, these data indicate that $\mathrm{ZnPP}$ can bind to telomerase complexes and/or associated components. While telomeric DNA does not appear to be a primary binding site of ZnPP per se, at least at prominent telomeres on metaphase chromosomes, the specific sites of interaction in the telomerase holoenzyme remain to be determined.

\section{4 | DISCUSSION}

The development of telomerase inhibitors for use in chemotherapy began shortly after the discovery of telomerase over three decades 
(A) Titration of complexes with ZnPP

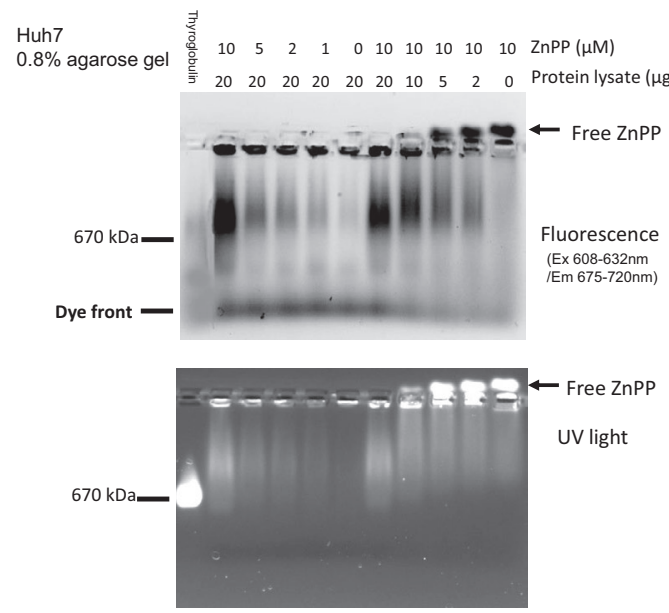

(B) ZnPP labelling of extracts $0.8 \%$ agarose gel

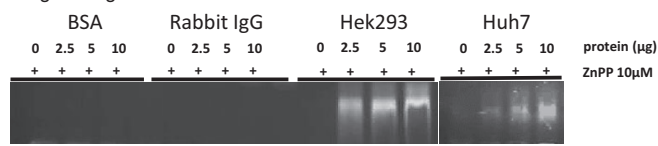

(C) ZnPP incubation of cultured cells $0.8 \%$ agarose gel
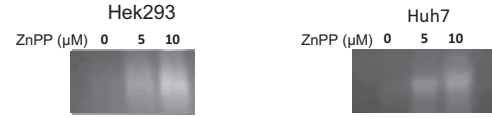

(D) Mobility shift with specific TERT antibody $0.8 \%$ agarose gel

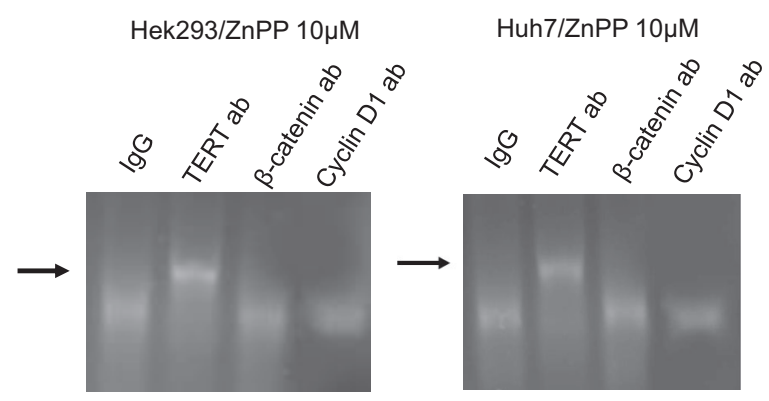

(E) Immunostaining of nitrocellulose blots for TERT and dyskerin

$0.8 \%$ agarose gel

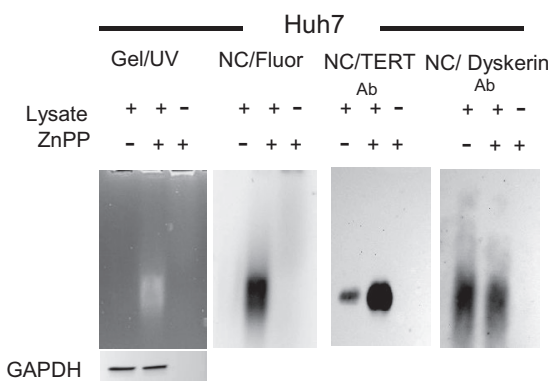

FIGURE 4 ZnPP fluorescent labeling of cellular extracts. (A) Titration of complexes with ZnPP. Cellular lysates as described in Methods were prepared from log phase Huh7 cells and incubated $1 \mathrm{~h}$ at RT with various concentrations of ZnPP. Mixtures were electrophoresed on non-denaturing $0.8 \%$ agarose gels and visualized fluorescently using red excitation 608-632 nm and emission 675-720 nm (upper panel) or broad band UV light (lower panel). (B) ZnPP labeling of extracts. ZnPP (10 $\mu$ M) was added to BSA, non-specific rabbit IgG, or cellular lysates from Hek293 and Huh7 at the indicated concentrations and incubated for $1 \mathrm{~h}$ at RT. Aliquots were then electrophoresed on $0.8 \%$ agarose gels and visualized under broad band UV light. (C) Labeling complexes with ZnPP in intact cells. ZnPP (0, 5, and $10 \mu \mathrm{M})$ was incubated with Hek293 and Huh7 cultured cells for 24 h, then enzymatic extracts were prepared, electrophoresed on $0.8 \%$ agarose gels, and visualized under broad band UV light. (D) ZnPP labeled complexes have mobility shift with anti-TERT antibodies. Enzymatic cellular extracts (10 $\mu$ g as protein) were prepared and labeled with $10 \mu \mathrm{M}$ ZnPP for $1 \mathrm{~h}$ at RT. The indicated antibodies were then added, and mixtures incubated at $4^{\circ} \mathrm{C}$ for $1 \mathrm{~h}$ prior to electrophoresis on $0.8 \%$ agarose gels and broad band UV light visualization. (ab = antibody). Arrow $=$ mobility shift of anti-TERT antibodies. (E) Immunostaining of ZnPP labeled complexes blotted to nitrocellulose. Lysates from Huh7 cells were incubated for $1 \mathrm{~h}$ at RT with $10 \mu \mathrm{M}$ ZnPP or vehicle control, then electrophoresed on agarose gels and diffusion-blotted onto nitrocellulose (NC). ZnPP complexes were visualized in gels by broad band UV and on NC blots by red fluorescence (wavelengths noted above). NC blots were then stained with specific anti-TERT or anti-dyskerin antibodies

ago. Telomerase inhibitors are cytotoxic to most tumor cells and promote telomere shortening and instability, DNA damage responses, DNA synthesis arrest, apoptosis, and other cellular senescence programs. ${ }^{6}$ Multiple telomerase inhibitors are in development and include competitive, non-competitive allosteric, and apparent DNA substrate inhibitors [see ${ }^{5}$ for a recent review].

A wide variety of planar aromatic macromolecules and porphyrins have also been shown to inhibit telomerase. ${ }^{46}$ These compounds are thought to stabilize hydrogen-bonded guanidine-tetrads (G4 complexes) that form in telomeric DNA and inhibit cyclic realignment of the DNA substrate with TERT during the enzyme cycle, thus reducing telomerase processivity. ${ }^{9,47}$ Functionally, synthetic porphyrins such as tetra-(N-methyl-4-pyridylporphyrin), (TMPyP4), were shown to cause cell growth arrest and apoptosis in neoplastic cells which demonstrated that $\mathrm{G} 4$ telomeric binding sites are useful targets for chemotherapy. ${ }^{10,48}$

In contrast to porphyrins, protoporphyrins have not been investigated for anti-telomerase behavior. Nevertheless, common MPPs such as FePP and ZnPP are known to bind selected oligomeric G-4 sequences in vitro, ${ }^{13,49}$ thus suggesting that they are capable of behaving like porphyrins for telomerase inhibition. Biologically, ZnPP and longacting conjugates such as ZnPP-polyethylene glycol have been studied as chemotherapeutic agents in vivo in rodent models for some time ${ }^{14}$ and promote tumor regression, both alone as well as synergistically with drugs such as cisplatin. ${ }^{15}$ Consequently, investigation of ZnPP effects and sites of action on telomerase activity is timely and important.

We first evaluated the effects of common MPPs on telomerase activity, cellular proliferation, DNA synthesis, and apoptosis. ZnPP 
(A)

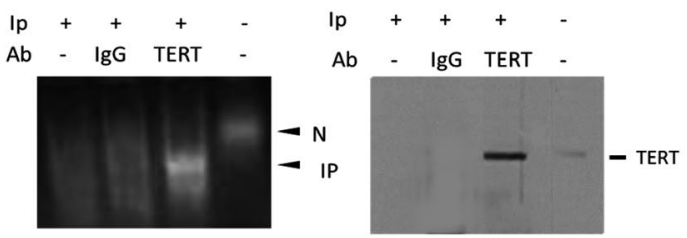

(B)

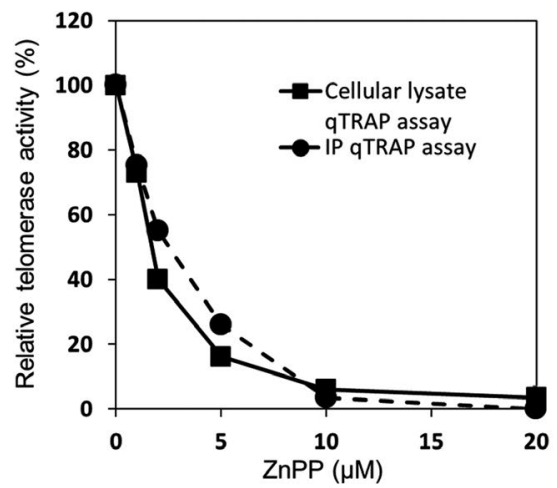

(C)

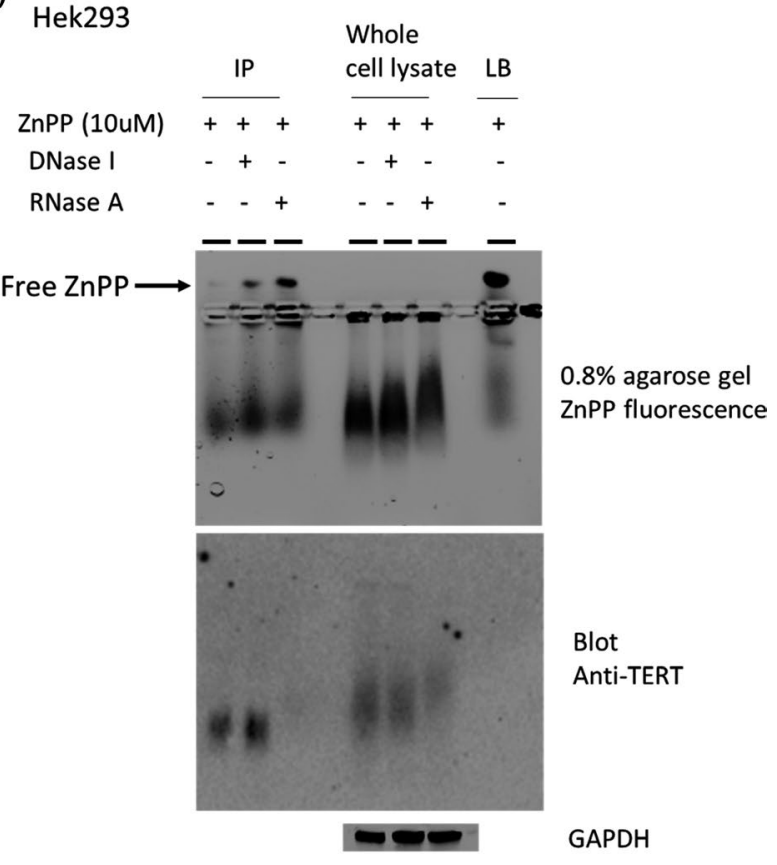

FIG URE 5 ZnPP inhibition and labeling of Immunoprecipitated TERT complexes. (A) ZnPP binds TERT IP complexes. Left panel: Immunoprecipitation was performed on lysates from Hek293 cells transfected with TERT and TERC plasmid constructs as described in the Methods. The IP were then re-suspended and incubated with ZnPP $(10 \mu \mathrm{M})$ for $1 \mathrm{~h}$ at RT prior to electrophoresis on $0.8 \%$ agarose gels and visualized under UV light. Right panel: Identical aliquots were also denatured for SDS-PAGE and evaluated on WB. (B) ZnPP inhibition of telomerase activity in cellular lysates and IP. TRAP assays were performed with IP or crude cellular lysates in the presence of various concentrations of ZnPP. No statistical difference of $\mathrm{ZnPPIC}_{50}$ of telomerase from either source (Table 2). (C) Nuclease digestion of ZnPP labeled complexes. Upper panel: Enzymatic extracts or IP (10 $\mu$ g protein) were treated with DNase 1 or RNase A ( $0.1 \mathrm{mg} / \mathrm{ml}$ at $4^{\circ} \mathrm{C}$ for $\left.1 \mathrm{~h}\right)$, then labeled with ZnPP $(10 \mu \mathrm{M})$ for $1 \mathrm{~h}$ at RT, before electrophoresis on $0.8 \%$ agarose gels and visualization of ZnPP by red fluorescence emission. Lower panel: Following electrophoresis, native proteins were transferred to NC by diffusion blotting and stained with antiTERT antibodies. For whole cell lysates, GAPDH was used as a loading control after lysates were electrophoresed on SDS-denaturing polyacrylamide gels and stained using WB methods ( $L B=$ lysis buffer); (IP= immunoprecipitate); $(\mathrm{N}=$ Native)

was the most potent MPP tested and down regulated TERT expression, arrested DNA synthesis, and promoted apoptosis in Huh7 hepatoma cells. In contrast, ZnPP was only minimally active in U2OS cells which contain no telomerase ${ }^{50}$ however, sensitivity could be acquired following TERT transfection. This suggests that telomerase interactions likely play a role in ZnPP anti-proliferative and apoptotic behavior. Downregulation of TERT was also accompanied by a reduction in cyclin D1 and $\beta$-catenin which are not only important markers of cellular proliferation and apoptosis per se, ${ }^{51}$ but they have known positive signaling relationships with telomerase. ${ }^{34,35}$ The data shown in Figure 1 also support earlier work that documented the specific anti-proliferative actions of $\mathrm{ZnPP}{ }^{23}$

ZnPP directly inhibited telomerase enzymatic activity in cellular lysates, IP, and intact cells in culture. This was verified by both TRAP and direct $\alpha{ }^{-32} \mathrm{P}$-dGTP extension assays with similar IC $\mathrm{C}_{50}$ (Table 1). To date, this is the first demonstration that ZnPP has direct antitelomerase activity and these findings support consideration of ZnPP or related MPP compounds for use in chemotherapeutic programs. These studies also lay groundwork for modeling and development of improved, anti-telomerase derivatives using a ZnPP-based model design.
$\mathrm{ZnPP}$ is a naturally occurring MPP and it inhibited telomerase at $\mathrm{EC}_{50}$ and $\mathrm{IC}_{50}$ concentrations of ca $5 \mu \mathrm{M}$ or less, close to levels that can be achieved physiologically. During normal heme synthesis in reticulocytes, ZnPP is produced at low levels $(0.5 \mu \mathrm{M})$, however, in times of iron deficiency it can rise to values of $5 \mu \mathrm{M}$ or higher, ${ }^{52}$ well within the effective anti-telomerase concentrations seen here. The $\mathrm{IC}_{50}$ of $\mathrm{ZnPP}$ (Table 2) is also close to the micromolar ranges of $\mathrm{IC}_{50}$ reported for other synthetic experimental porphyrins. ${ }^{6,46}$

The major components of the telomerase holoenzyme complex include TERT, dyskerin, p23, Hsp90, TERC and telomerase-associated protein. ${ }^{53}$ TERT is absent from telomeres until it is assembled into telomerase holoenzyme and then recruited to selective chromosomal telomeric sites at the start of S phase. ${ }^{45,54}$ Telomere addition is known to be coupled to DNA synthesis and the processes likely occur sequentially in neoplastic cells. ${ }^{45}$ While the latter interaction may very well account for the profound depression of DNA synthesis in neoplastic cells, we cannot rule out the possibility that ZnPP also has a direct effect on DNA polymerases.

With the structural and functional characteristics of telomerase in mind, we investigated whether ZnPP can bind at or near 


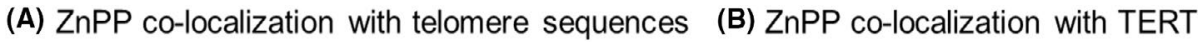

S phase

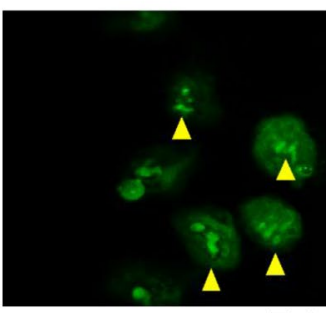

Telomere stain

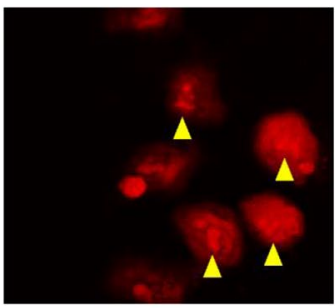

ZnPP stain
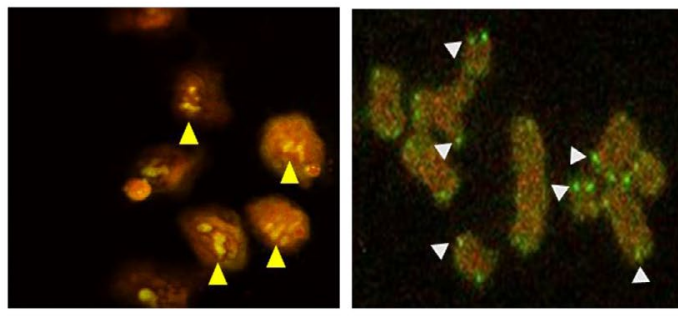

Merged
Metaphase
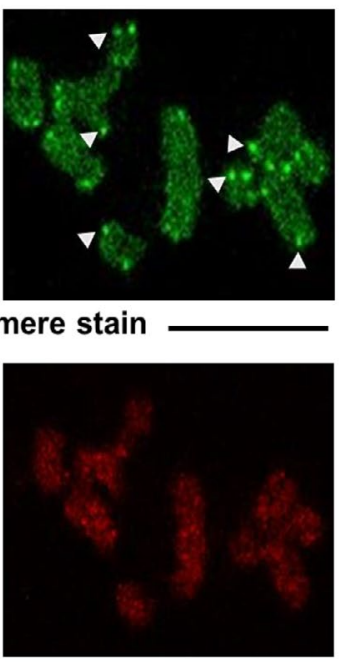

$S$ phase

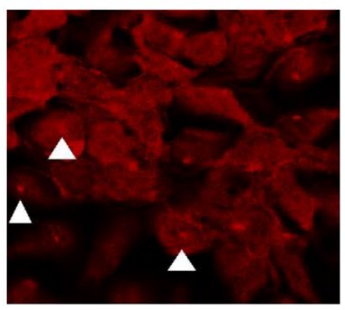

ZnPP Stain
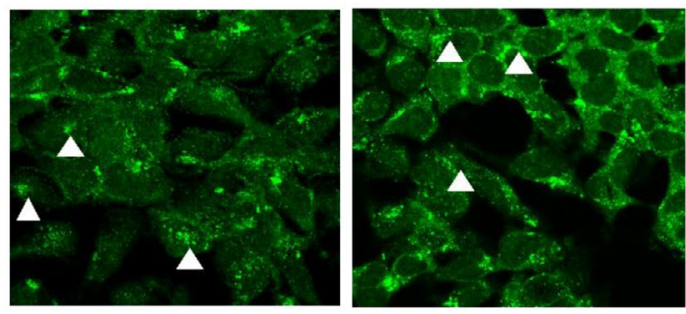

Anti-TERT
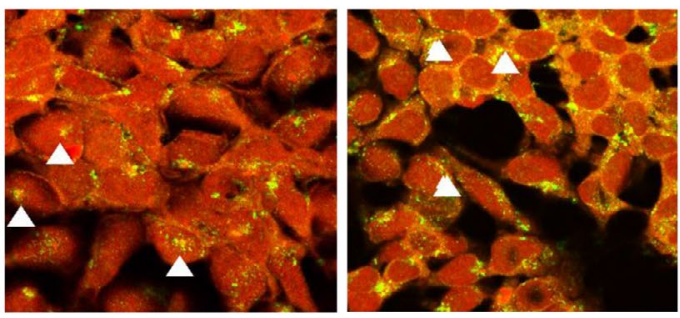

Merged

FIGURE 6 Fluorescence co-localization of ZnPP with telomeres and TERT. (A) Co-localization of ZnPP and telomeres. Synchronized S phase Huh7 cells (left panels) and metaphase chromosomal spreads (right panels) were prepared as described in Methods. Fixed preparations were reacted with the specific fluorescent telomere probe TelC-Alexa488 and $10 \mu \mathrm{M}$ ZnPP. Confocal microscopy used Alexa Fluor 488 (green filter) or Alexa Fluor 568 (red filter) to visualize telomeres or ZnPP, respectively, and merged sites (yellow). Yellow arrows (left panels) show co-localization of telomeres and ZnPP. White arrows, (right panels) show localization of telomeres which do not co-localize with ZnPP. (B) Colocalization of ZnPP and TERT in synchronized S phase (left panels) and unsynchronized log phase (right panels). Huh7 cells were incubated with $10 \mu \mathrm{M}$ ZnPP after labeling with anti-TERT antibodies. Confocal microscopy was then conducted using Alexa Fluor 488 (green filter, anti-TERT) or Alexa Fluor 568 (red filter, ZnPP) fluorochromes to visualize TERT or ZnPP, respectively, and merged sites (yellow). Arrows depict example sites of co-localization of ZnPP and TERT in the nuclei of S phase cells (left panels) or cytoplasm/perinuclear regions of unsynchronized semi-confluent cells (right panels)

holoenzyme complexes. On non-denaturing agarose gels, ZnPP specifically bound to high molecular weight complexes [>670 kD] in cellular extracts. The binding of anti-TERT antibodies to ZnPPlabeled complexes resulted in an upward mobility shift, thus demonstrating that the complexes contained TERT. Both TERT and dyskerin were identified in ZnPP labeled complexes by native agarose gel immunoblot analysis. Consequently, ZnPP can bind native high molecular weight complexes that contain components of the telomerase holoenzyme.

As might be expected, IP showed faster mobility than complexes from crude lysates indicating a smaller, immunopurified preparation. DNase 1 (mostly selective for DS DNA) had minimal effects on ZnPP binding while RNase A digestion led to decreased complex mobility, decreased ZnPP binding, and increased free
ZnPP. Moreover, RNase A digestion caused loss of TERT from the complexes, a result that might be anticipated since TERC binds TERT in the ribonuclear protein complex and RNase A treatment disrupts holoenzyme structure. ${ }^{41}$ Collectively, these data demonstrate that ZnPP binds ribonuclear protein complexes containing TERT, however, identification of the definitive binding sites requires further study.

ZnPP has been reported to bind some selected G-4 structures as well as oligomeric telomere sequences in vitro, ${ }^{13}$ thus, considerable heterogeneity of ZnPP binding should be expected. G-rich DNA sequences likely modulate DNA structure-regulatory activities at sites such as gene promoters for enzyme systems in addition to telomerase. ${ }^{55}$ Consequently, we cannot yet account for how other ZnPP targeted G4 sites may contribute to our results here. 
Confocal immunohistochemical experiments demonstrated that ZnPP binds at or near telomere/telomerase complexes in S phase cells, which is the only time in the cell cycle when telomerase is found at telomeres. ${ }^{45}$ Surprisingly, telomere rich sites on metaphase chromosomal ends did not overtly bind ZnPP (Figure 6A). Consequently, ZnPP interactions with telomerase complexes appear more complex than just telomere G4 binding and may depend on $\mathrm{S}$ phase chromatin structure, site accessibility, and composition of the complexes. Furthermore, these data suggest there may be a primary interaction of ZnPP with TERT or closely associated proteins since there was co-localization of TERT and ZnPP in cytoplasmic as well as nuclear locations (Figure 6B). Interestingly, earlier work showed that ZnPP and FePP could directly bind other reverse transcriptases such as $\mathrm{HIV}^{56}$ also supporting a direct interaction of ZnPP and TERT. More studies characterizing the site(s) of ZnPP binding and kinetics of telomerase inhibition are necessary. Overall, the ability of ZnPP to avidly bind telomere sites in S-phased cells in situ strongly suggests it can impact telomerase activity where and when telomerase is active intracellularly as supported by the kinetic and immunoblot experiments.

Aside from telomerase, other cellular sites, both nuclear and cytoplasmic, have been proposed to be responsible for the pro-apoptotic qualities of ZnPP. Since ZnPP is both a transcriptional inducer for heme oxygenase- $1(\mathrm{HO}-1)^{57}$ and a competitive $\mathrm{HO}-1$ inhibitor, ${ }^{58}$ earlier studies attributed many ZnPP mechanisms to HO-1 antagonism. Presently it is not known whether ZnPP actions on the telomerase system are impacted by HO-1 antagonism and this concept requires further study. ZnPP also inhibited transcriptional promoter sites for cyclin D $1^{23}$ and attenuated Wnt/ $\beta$-catenin expression leading to increased apoptosis. ${ }^{59}$ Our proliferation studies support these findings, yet it is not yet clear whether telomerase inhibition may impact these other pathways.

\section{5 | CONCLUSIONS}

In summary, our data indicate that ZnPP interacts with the telomerase enzyme system at two major regulatory points: (1) downregulation of TERT and (2) direct inhibition of telomerase enzymatic activity. Concomitantly, ZnPP attenuates DNA synthesis and cellular proliferation while promoting apoptosis. Structurally, ZnPP binds to TERT containing ribonuclear protein complexes and co-localizes with a subset of nuclear telomeres that likely contain holoenzyme complexes. Our findings support the use of ZnPP and potentially the development of other synthetic or natural protoporphyrins for use as chemotherapeutic agents in the treatment of neoplastic disease. Ongoing further experiments aim to characterize the binding and inhibition sites of ZnPP in the telomerase complexes.

\section{ACKNOWLEDGEMENT}

None.

\section{CONFLICT OF INTEREST}

The authors declare there are no conflicts of interests.

\section{AUTHORS' CONTRIBUTIONS}

Zhu, Tran, Meier, and Schmidt participated in research design. Zhu, Tran, Mathahs, Albert, and Fink conducted experiments. Zhu, Tran, Moninger, Meier, Li, and Schmidt contributed new reagents or analytic tools. Zhu, Fink, Albert, Meier, and Schmidt performed data analysis. Zhu, Tran, and Schmidt wrote or contributed to the writing of the manuscript.

\section{ETHICS STATEMENT}

This paper is the original and authentic work of the authors. All authors read and approved the final manuscript.

\section{DATA AVAILABILITY STATEMENT}

All data underlying the results are freely available as part of the article and no additional source data are required.

\section{ORCID}

Warren N. Schmidt (D) https://orcid.org/0000-0003-2893-6014

\section{REFERENCES}

1. Jafri MA, Ansari SA, Alqahtani MH, Shay JW. Roles of telomeres and telomerase in cancer, and advances in telomerase-targeted therapies. Genome Med. 2016;8:69.

2. Kubicka S, Rudolph KL, Hanke M, et al. Hepatocellular carcinoma in Germany: a retrospective epidemiological study from a lowendemic area. Liver. 2000;20:312-318.

3. Bryan TM, Englezou A, Dalla-Pozza L, Dunham MA, Reddel RR. Evidence for an alternative mechanism for maintaining telomere length in human tumors and tumor-derived cell lines. Nat Med. 1997;3:1271-1274.

4. Djojosubroto MW, Chin AC, Go N, et al. Telomerase antagonists GRN163 and GRN163L inhibit tumor growth and increase chemosensitivity of human hepatoma. Hepatology. 2005;42:1127-1136.

5. Ivancich $M$, Schrank Z, Wojdyla L, et al. Treating cancer by targeting telomeres and telomerase. Antioxidants. 2017;6(1):15.

6. De Cian A, Lacroix L, Douarre C, et al. Targeting telomeres and telomerase. Biochimie. 2008;90:131-155.

7. Shi DF, Wheelhouse RT, Sun DY, Hurley LH. Quadruplex-interactive agents as telomerase inhibitors: Synthesis of porphyrins and structure-activity relationship for the inhibition of telomerase. $J$ Med Chem. 2001;44:4509-4523.

8. Huppert JL. Four-stranded nucleic acids: structure, function and targeting of G-quadruplexes. Chem Soc Rev. 2008;37:1375-1384.

9. Dixon IM, Lopez F, Esteve JP, et al. Porphyrin derivatives for telomere binding and telomerase inhibition. ChemBioChem. 2005;6:123-132.

10. Shammas MA, Shmookler Reis RJ, Akiyama M, et al. Telomerase inhibition and cell growth arrest by G-quadruplex interactive agent in multiple myeloma. Mol Cancer Ther. 2003;2:825-833.

11. Sen D, Poon LC. RNA and DNA complexes with hemin [Fe(III) heme] are efficient peroxidases and peroxygenases: how do they do it and what does it mean? Crit Rev Biochem Mol Biol. 2011;46:478-492.

12. Saito K, Tai H, Hemmi H, Kobayashi N, Yamamoto Y. Interaction between the heme and a G-quartet in a heme-DNA complex. Inorg Chem. 2012;51:8168-8176. 
13. Zhang Z, Sharon E, Freeman R, Liu X, Willner I. Fluorescence detection of DNA, adenosine-5'-triphosphate (ATP), and telomerase activity by zinc(II)-protoporphyrin IX/G-quadruplex labels. Anal Chem. 2012b;84:4789-4797.

14. Fang J, Sawa T, Akaike T, Greish K, Maeda H. Enhancement of chemotherapeutic response of tumor cells by a heme oxygenase inhibitor, pegylated zinc protoporphyrin. Int J Cancer. 2004;109:1-8.

15. Liu Y-S, Li H-S, Qi D-F, et al. Zinc protoporphyrin IX enhances chemotherapeutic response of hepatoma cells to cisplatin. World J Gastroentero. 2014;20:8572-8582.

16. Zhu Z, Wilson AT, Luxon BA, et al. Biliverdin inhibits hepatitis $C$ virus nonstructural $3 / 4 A$ protease activity: mechanism for the antiviral effects of heme oxygenase? Hepatology. 2010a;52:1897-1905

17. Lohmann V, Korner F, Koch JO, Herian U, Theilmann L, Bartenschlager R. Replication of subgenomic hepatitis C virus RNAs in a hepatoma cell line. Science. 1999;285:110-113.

18. Zhu Z, Wilson AT, Mathahs MM, et al. Heme oxygenase-1 suppresses hepatitis $C$ virus replication and increases resistance of hepatocytes to oxidant injury. Hepatology. 2008;48:1430-1439.

19. Meyerson M, Counter CM, Eaton EN, et al. hEST2, the putative human telomerase catalytic subunit gene, is up-regulated in tumor cells and during immortalization. Cell. 1997;90:785-795.

20. Fu D, Collins K. Distinct biogenesis pathways for human telomerase RNA and H/ACA small nucleolar RNAs. Mol Cell. 2003;11:1361-1372.

21. Zhu Z, Tran H, Mathahs MM, Moninger TO, Schmidt WN. HCV induces telomerase reverse transcriptase, increases its catalytic activity, and promotes caspase degradation in infected human hepatocytes. PLOS ONE. 2017;12:e0166853.

22. Hu K, Zhu Z, Mathahs MM, et al. Metalloprotoporphyrin inhibition of HCV NS3-4A protease: structure-activity relationships. Drug Des Devel Ther. 2020;14:757-771.

23. La P, Fernando AP, Wang Z, et al. Zinc protoporphyrin regulates cyclin D1 expression independent of heme oxygenase inhibition. $J$ Biol Chem. 2009;284:36302-36311.

24. Zhu Z, Mathahs MM, Schmidt WN. Restoration of type I interferon expression by heme and related tetrapyrroles through inhibition of NS3/4A protease. J Infect Dis. 2013;208:1653-1663.

25. Zhu ZW, Wilson AT, Gopalakrishna K, Brown KE, Luxon BA, Schmidt WN. Hepatitis $C$ virus core protein enhances telomerase activity in Huh7 cells. J Med Virol. 2010b;82:239-248.

26. Tomlinson CG, Sasaki N, Jurczyluk J, Bryan TM, Cohen SB. Quantitative assays for measuring human telomerase activity and DNA binding properties. Methods. 2017;114:85-95.

27. Kim R. Native agarose gel electrophoresis of multiprotein complexes. Cold Spring Harb Protoc. 2011;2011:884-887.

28. Abdalla MY, Britigan BE, Wen F, et al. Down-regulation of heme oxygenase- 1 by hepatitis $C$ virus infection in vivo and by the in vitro expression of hepatitis $C$ core protein. J Infect Dis. 2004;190:1109-1118.

29. Acharya S, Kaul Z, Gocha AS, et al. Association of BLM and BRCA1 during telomere maintenance in ALT cells. PLOS ONE. 2014;9:e103819.

30. Harding SD, Sharman JL, Faccenda E, et al. The IUPHAR/BPS Guide to pharmacology in 2018: updates and expansion to encompass the new guide to immunopharmacology. Nucleic Acids Res. 2018;46:D1091-D1106.

31. Alexander SP, Fabbro D, Kelly E, et al. THE concise guide to pharmacology 2021/22: catalytic receptors. Br J Pharmacol. 2021;178(Suppl 1):S264-S312.

32. Pascolo E, Wenz C, Lingner J, et al. Mechanism of human telomerase inhibition by BIBR1532, a synthetic, non-nucleosidic drug candidate. J Biol Chem. 2002;277:15566-15572.

33. Damm K, Hemmann U, Garin-Chesa P, et al. A highly selective telomerase inhibitor limiting human cancer cell proliferation. Embo J. 2001;20:6958-6968.
34. Li J, Huang X, Xie X, Wang J, Duan M. Human telomerase reverse transcriptase regulates cyclin D1 and G1/S phase transition in laryngeal squamous carcinoma. Acta Otolaryngol. 2011;131:546-551.

35. Zhang $\mathrm{Y}$, Toh L, Lau $\mathrm{P}$, Wang $X$. Human telomerase reverse transcriptase (hTERT) is a novel target of the Wnt/beta-catenin pathway in human cancer. J Biol Chem. 2012a;287:32494-32511.

36. De Cian A, Cristofari G, Reichenbach P, et al. Reevaluation of telomerase inhibition by quadruplex ligands and their mechanisms of action. Proc Natl Acad Sci USA. 2007;104:17347-17352.

37. Lamola AA. Fluorescence methods in the diagnosis and management of diseases of tetrapyrrole metabolism. J Invest Dermatol. 1981;77:114-121.

38. Tong A-J, Liu L, Liu L, Li L-D, Huie CW. Solid-substrate roomtemperature phosphorescence study on zinc(II) and tin(IV) protoporphyrins and their interaction with DNA. Fresenius J Anal Chem. 2001;370:1023-1028.

39. Yang G, Nguyen X, Ou J, Rekulapelli P, Stevenson DK, Dennery PA. Unique effects of zinc protoporphyrin on $\mathrm{HO}-1$ induction and apoptosis. Blood. 2001;97:1306-1313.

40. Gardano L, Holland L, Oulton R, Le Bihan T, Harrington L. Native gel electrophoresis of human telomerase distinguishes active complexes with or without dyskerin. Nucleic Acids Res. 2012;40:e36.

41. Venteicher AS, Meng ZJ, Mason PJ, Veenstra TD, Artandi SE. Identification of ATPases pontin and reptin as telomerase components essential for holoenzyme assembly. Cell. 2008;132:945-957.

42. Diehl JA, Yang W, Rimerman RA, Xiao H, Emili A. Hsc70 regulates accumulation of cyclin D1 and cyclin D1-dependent protein kinase. Mol Cell Biol. 2003;23:1764-1774.

43. Gerlach JP, Emmink BL, Nojima H, Kranenburg O, Maurice MM. Wnt signalling induces accumulation of phosphorylated beta-catenin in two distinct cytosolic complexes. Open Biol. 2014;4:140120.

44. Venteicher AS, Abreu EB, Meng Z, et al. A human telomerase holoenzyme protein required for Cajal body localization and telomere synthesis. Science (New York, NY). 2009;323:644-648.

45. Tomlinson RL, Ziegler TD, Supakorndej T, Terns RM, Terns MP. Cell cycle-regulated trafficking of human telomerase to telomeres. Mol Biol Cell. 2006;17:955-965.

46. Cao Q, Li Y, Freisinger E, Qin PZ, Sigel RKO, Mao Z-W. G-quadruplex DNA targeted metal complexes acting as potential anticancer drugs. Inorg Chem Front. 2017;4:10-32.

47. Sun D, Thompson B, Cathers BE, et al. Inhibition of human telomerase by a G-quadruplex-interactive compound. J Med Chem. 1997;40:2113-2116.

48. Izbicka E, Wheelhouse RT, Raymond E, et al. Effects of cationic porphyrins as G-quadruplex interactive agents in human tumor cells. Can Res. 1999;59:639-644.

49. Gray LT, Puig Lombardi E, Verga D, et al. G-quadruplexes sequester free heme in living cells. Cell Chem Biol. 2019;26:1681-1691. e1685.

50. Alawi F, Lin P. Dyskerin is required for tumor cell growth through mechanisms that are independent of its role in telomerase and only partially related to its function in precursor rRNA processing. Mol Carcinog. 2011;50:334-345.

51. Veeramachaneni NK, Kubokura $\mathrm{H}$, Lin L, et al. Down-regulation of beta catenin inhibits the growth of esophageal carcinoma cells. $J$ Thorac Cardiovasc Surg. 2004;127:92-98.

52. Iyer JK, Shi L, Shankar AH, Sullivan DJ Jr. Zinc protoporphyrin IX binds heme crystals to inhibit the process of crystallization in Plasmodium falciparum. Mol Med. 2003;9:175-182.

53. Wyatt HDM, West SC, Beattie TL. InTERTpreting telomerase structure and function. Nucleic Acids Res. 2010;38:5609-5622.

54. Vogan JM, Collins K. Dynamics of Human Telomerase Holoenzyme Assembly and Subunit Exchange across the Cell Cycle. J Biol Chem. 2015;290:21320-21335. 
55. Huppert JL, Balasubramanian S. Prevalence of quadruplexes in the human genome. Nucleic Acids Res. 2005;33:2908-2916.

56. Argyris EG, Vanderkooi JM, Paterson Y. Mutagenesis of key residues identifies the connection subdomain of HIV-1 reverse transcriptase as the site of inhibition by heme. Eur J Biochem. 2001;268:925-931.

57. Kwok SCM. Zinc protoporphyrin upregulates heme oxygenase- 1 in PC-3 cells via the stress response pathway. Int J Cell Biol. 2013;2013:162094.

58. Maines MD. Zinc. protoporphyrin is a selective inhibitor of heme oxygenase activity in the neonatal rat. Biochem Biophys Acta. 1981;673:339-350.

59. Wang S, Hannafon BN, Lind SE, Ding W-Q. Zinc protoporphyrin suppresses beta-catenin protein expression in human cancer cells: the potential involvement of lysosome-mediated degradation. PLoS ONE. 2015;10:e0127413.

\section{SUPPORTING INFORMATION}

Additional supporting information may be found in the online version of the article at the publisher's website.

How to cite this article: Zhu Z, Tran H, Mathahs MM, et al. Zinc protoporphyrin binding to telomerase complexes and inhibition of telomerase activity. Pharmacol Res Perspect. 2021;9:e00882. doi:10.1002/prp2.882 